\title{
Swelling of 9 polymers commonly employed for solvent-resistant nanofiltration membranes: a comprehensive dataset
}

Emiel J. Kappert ${ }^{1, \mathrm{a}, *}$, Michiel J.T. Raaijmakers ${ }^{1, \mathrm{~b}}$, Kristianne Tempelman ${ }^{1}$, F. Petrus Cuperus ${ }^{2}$, Wojciech Ogieglo $^{1, \mathrm{c}}$, Nieck E. Benes ${ }^{1}$

\section{Graphical Abstract}

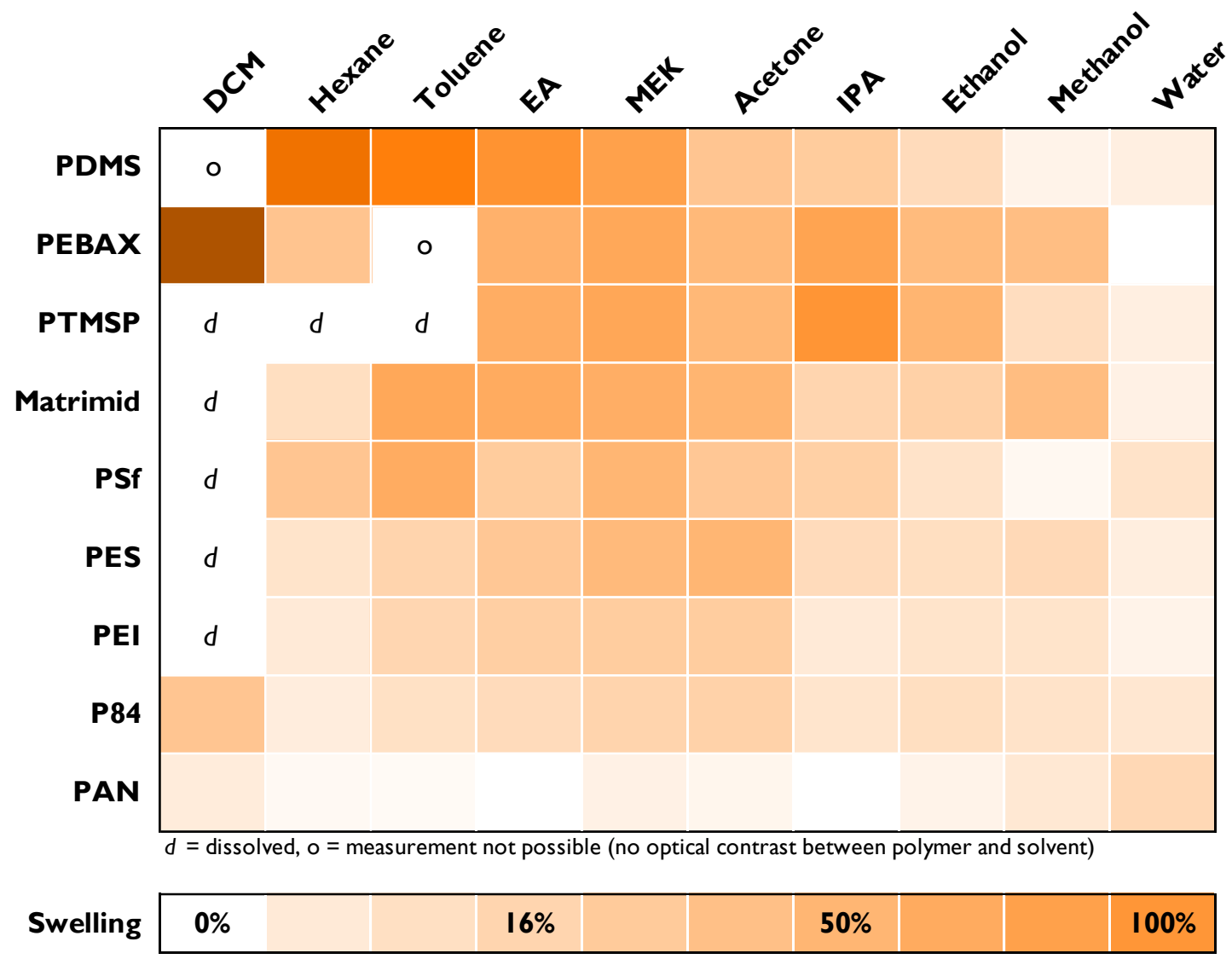

\footnotetext{
${ }^{1}$ Films in Fluids, Department of Science and Technology, MESA ${ }^{+}$Institute for Nanotechnology, University of Twente, P.O. Box 217, 7500 AE Enschede, The Netherlands. ${ }^{2}$ SolSep B.V. Robust Membrane Technologies, St. Eustatius 65, NL-7333 NW Apeldoorn, Netherlands. ${ }^{\text {a }}$ Present address: BASF SE, D-67056 Ludwigshafen, Germany. ${ }^{b}$ Present address: AkzoNobel Specialty Chemicals, Zutphenseweg 10, P.O. Box 10, 7400AA Deventer, The Netherlands. ${ }^{c}$ Present address: Functional Polymer Membranes Group, Advanced Membranes and Porous Materials Center, Division of Physical Sciences and Engineering, King Abdullah University of Science and Technology (KAUST), Thuwal, Jeddah 23955-6900, Kingdom of Saudi Arabia.
} 


\section{Abstract}

The presence of a solvent in a dense polymeric nanofiltration membrane layer can affect the macromolecular dynamics of the polymer material and the mobility of the solvent penetrant molecules. In addition, even the affinity of the swollen material for the solvent molecules can be distinct from that of the pure polymer material. These effects can substantially affect the membrane's performance. This paper provides sorption and swelling data of 9 thin polymer films that are commonly used for organic solvent nanofiltration (P84, Matrimid, PEI, PAN, PES, PSf, PEBAX, PTMSP, PDMS) swollen by 10 common solvents (hexane, toluene, dichloromethane, ethyl acetate, methyl ethyl ketone, acetone, isopropanol, ethanol, methanol, water). The paper describes the swelling dynamics during short-term solvent exposure $(0-8 \mathrm{~h})$, assesses the stability upon long-term solvent exposure (up to 2 months), and provides quantitative data on the solvent volume fractions inside the swollen layers. Among the surprising findings are the glubbery behavior of PTMSP and the completely different response of PES and PSf to toluene exposure. The results of this work demonstrate three crucial findings relevant to organic solvent nanofiltration membranes and other applications:

1. For many polymers, the swelling changes over long timescales of up to 2 months and longer. Results obtained on short timescales do however not always allow for direct extrapolation to longer time scales.

2. Structural similarity of polymers does not guarantee similar swelling behavior;

3. Swelling behavior of solvents cannot be solely explained by classifying solvents into aprotic, polar aprotic and polar protic solvents.

The results of this work can aid in constructing transport models and can help predicting polymer-solvent compatibility and membrane performance in OSN applications.

Keywords: Organic solvent nanofiltration (OSN), solvent resistant nanofiltration (SRNF), polymer swelling dynamics, in situ spectroscopic ellipsometry 


\section{Introduction}

Organic solvent nanofiltration (OSN) membranes separate solvents from solutes based on the difference in their permeability through the membrane [1,2]. Solvent permeation through dense nanofiltration membranes can be described by the combined contributions of the dissolution of the solvent into the polymer and its diffusion through the polymer layer. The presence of the solvent in the polymer matrix can result in changes in the properties of the polymer layer, of which the most evident change is the swelling of the polymer matrix. In membrane applications, the swelling of the polymer matrix can result in a decreased retention of small solutes [3], an increased diffusion coefficient for the permeating components [4], plasticization [5-8] and/or pore collapse and mechanical instabilities [1,9].

To study membrane swelling, it is important to distinguish between the swelling or dilation of the material on one hand and the solvent (mass) uptake in the layer on the other hand. In the field of membrane science, the term swelling is often used synonymously with mass uptake, whereas in the field of polymer science swelling typically refers to dilation. These two terms have to be clearly distinguished because they do not necessarily represent the same phenomenon. For instance, a glassy polymer containing excess free volume may take up solvent without displaying significant dilation. In this manuscript, we adopt the term swelling to describe the dimensional dilation of the layer and solvent uptake to describe the increase in the amount of solvent inside the layer.

Typically gravimetric methods are employed to determine the solvent mass uptake by a polymer [10-14]. Alternatively, measurement of changes in the size of the material can be used to calculate the dilation [14-17]. However, neither of these two metrics (solvent mass uptake nor dilation) can be used to accurately calculate the solvent concentration inside a layer without making a series of assumptions, such as the molar volume of the solvent in the polymer. Accurate data on the solvent concentration inside a thin film is of crucial importance, since it is directly correlated to the chemical potential of the solvent - the thermodynamic driving force for solvent-induced changes. Therefore, it is important to simultaneously determine the swelling of and solvent uptake by a layer. Better understanding of the swelling dynamics would allow for selection of suitable polymer-solvent combinations and prediction of long-term changes in membrane properties.

Characterization of thin membrane films is further complicated because the behavior of a thin film of sub-micron thickness can be different from that of a bulk polymer that is measured in the common swelling methods [18-20]. Firstly, the confinement of a thin 
membrane film to a substrate restricts the swelling to one dimension, causing the (elastic) strain energy contributions for a given volumetric change to be higher for a thin film as compared to a bulk polymer that can swell in three dimensions (see Supplementary Information, eqn. 25). Secondly, thin films may behave systematically different from bulk samples, for instance because of nano-confinement effects (e.g., interface effects, polymer chain relaxation dynamics, residual stress) [21] or different time scales for diffusion as a result of the different diffusion length $[22,23]$.

To mimic the conditions to which a membrane is exposed, thin films that are confined to a substrate need to be studied. Measurements on the layers need to be performed in the solvent (in situ) and must address both the short as well as the long-term behavior, as changes in membrane performance may manifest themselves promptly as well as gradually over time. Most studies that (partially) satisfy these measurement parameters and deal with swollen glassy films or membranes have been performed on model systems (e.g., PS [24,25], PMMA [26]) or have used solvent vapors instead of liquids (e.g., [15,27,28]); these rarely represent the polymers and conditions used in OSN applications. In this context, we aim at providing essential data on solvent-induced swelling, dynamics, and stability of relevant glassy polymers with a thin film geometry.

In this paper, we assess the swelling and solvent uptake of 9 typical hydrophobic membrane polymers in 10 commonly used solvents. The polymers poly(acrylonitrile) (PAN), poly(sulfone) (PSf), and poly(ether sulfone) (PES) have been selected because of their use in membrane supports. Polydimethylsiloxane (PDMS), poly(ether imide) (PEI), poly(ether block amide) (trade name: PEBAX), poly(imide) (P84 and Matrimid), and poly[1(trimethylsilyl)-1-propyne) (PTMSP) have been selected because of their use in separation layers. The 10 solvents include apolar (hexane, toluene), polar protic (water, methanol $(\mathrm{MeOH})$, ethanol (EtOH), isopropanol (IPA)) and polar aprotic (acetone, methyl-ethyl ketone (MEK), ethyl acetate (EA)) solvents. The final solvent studied is DCM, which is on the border between apolar and polar aprotic [29]. Since pure solvents were used, the solvent activity was equal to 1 in all measurements. The measurements were performed in situ with a high temporal resolution for the first 8 hours following solvent exposure to study the swelling dynamics and subsequently over the course of 2 months with intermittent storage in the solvent to study the long-term effects of solvent exposure to the polymer layers.

For every polymer-solvent combination, the thickness (as a measure for swelling) and the refractive index (reflecting the solvent uptake) of the swollen layer were measured independently by spectroscopic ellipsometry, resulting in a vast dataset of well over 3000 
measurement entries. The short-term measurements give clear insight in the swelling dynamics. The long-term measurements reflect the changes to the polymer upon exposure to the solvent for up to 2 months. These data allow for a more accurate quantification of the swelling degree of the layers and for studying the stability of polymers in contact with solvents. The presented data form a comprehensive dataset that allows for close investigation of specific polymers-solvent combinations. We believe that this data set aids in increasing the fundamental understanding of OSN membranes and can find use as a practical guide for choosing an appropriate membrane system for a given OSN process.

\section{Theoretical considerations}

\subsection{Determining swelling and solvent uptake by ellipsometry}

Spectroscopic ellipsometry is an optical measurement technique that allows for determining the thickness $d$ and the refractive index $n$ of a layer from the changes in the polarization state of light reflected from the sample, given by $\Psi$ and $\Delta$. The refractive index is a measure of the density of the layer and as such can be used to determine the amount of solvent inside a swollen membrane layer. The layer thickness and refractive index can be determined independently by fitting an optical model to the measured $\Psi$ and $\Delta$-data. By incorporating the refractive index of the solvent into the optical model, the thickness and refractive index of the swollen film can be tracked in situ. Figure 1 shows a schematic representation of the sample cell that is used for the measurements. A detailed summary of ellipsometry for studying in situ swelling is given in reference [30].

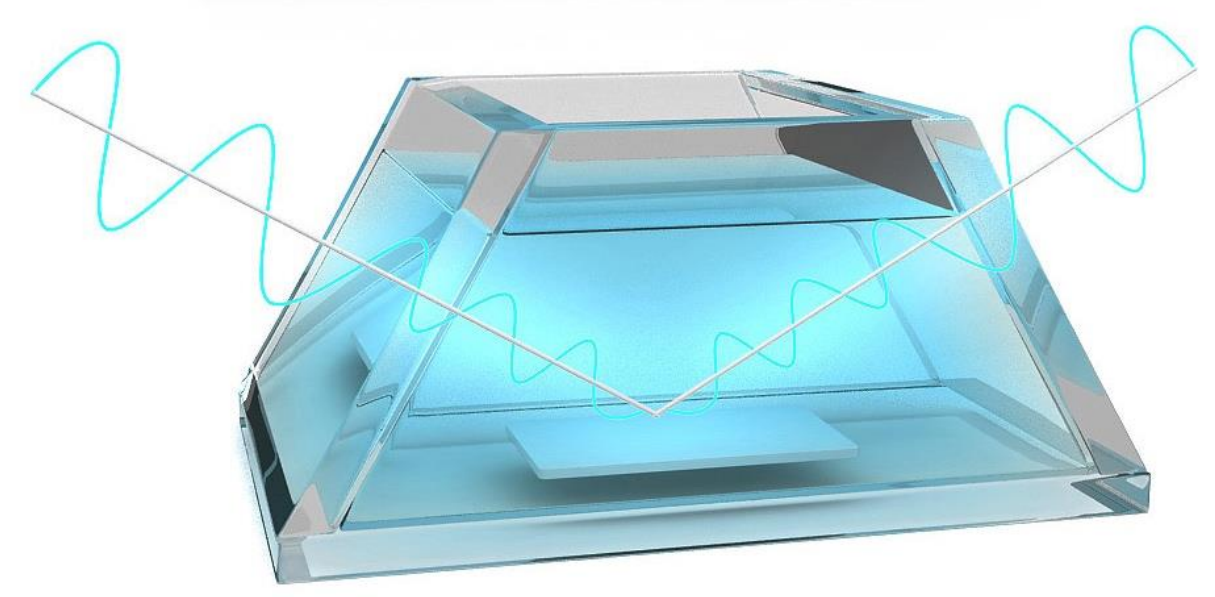

Figure 1: Schematic drawing of the measurement cell for in-situ spectroscopic ellipsometry

\subsection{Definitions and calculations}

\subsubsection{Swelling degree}


In this manuscript, the swelling degree $S D$ (-) equals the swelling factor $S F(-)$ minus 1 and is calculated from the ratio of the thicknesses of the swollen and dry film, by:

$$
S D=S F-1=\frac{d_{\text {swollen }}}{d_{\text {dry }}}-1
$$

\subsubsection{Normalized refractive index}

The refractive index of the swollen layers is normalized to the refractive indices of the dry layer and the solvent, by:

$$
n_{\text {normalized }}=\frac{n_{\text {swollen }}-n_{\text {solvent }}}{n_{\text {dry polymer }}-n_{\text {solvent }}}
$$

The normalized refractive index is a measure for the optical properties of the layer: a value of 1 indicates that the "swollen" material has a refractive index identical to that of the pure polymer, whereas a value of 0 would indicate that the layer has attained the refractive index of the solvent (typically implying dissolution). $n_{\text {normalized }}$ can exceed the value of 1 when any excess free volume in the material is filled by the solvent $\left(n_{\text {solvent }}>n_{\text {void }}=1\right)$. It must be noted that the normalized refractive index does not correlate linearly with the solvent volume fraction in the layer (see Section 2.4).

\subsubsection{Solvent volume fraction}

For polymers without an excess free volume (i.e., rubbery polymers), the volume fractions of a solvent in a swollen film $\Phi_{\mathrm{s}}(-)$ and of the polymer in a swollen film $\Phi_{\mathrm{p}}(-)$ can be directly calculated from the swelling factor by:

$$
\Phi_{\mathrm{s}}=1-\Phi_{\mathrm{p}}=1-\frac{1}{S F}
$$

This approach assumes no changes in the molar volumes of the solvent and the polymer and that the solvent-induced volume changes for a thin supported film are forced to occur only in the direction perpendicular to a substrate due to the confinement in the lateral plane.

For systems where volume additivity holds (mostly rubbery polymers with relatively weakly interacting penetrants) the dilation (Equation 3) and EMA (Equation 5) approaches are known to agree within experimental errors and yield accurate results [15,31-35]. For glassy polymers, which possess non-equilibrium excess free volume (EFV), these approaches do not agree [36]. For more details on calculating the solvent fraction inside a swollen glassy polymer, the reader is referred to reference [36]. 


\subsection{Thermodynamics of polymer swelling and their application to membrane transport}

In this section, the theoretical background behind the swelling of polymers and the relation between the swelling and membrane performance are described. The aim of the present paper is to provide the data that is crucial for applying all these theories.

\subsubsection{Swelling of rubbery polymers}

Amorphous polymer systems in equilibrium with a solvent are widely described by the Flory-Huggins theory [37]. This theory employs statistical thermodynamics to derive an expression that describes the sorption equilibrium between a polymer and a solvent. Under the assumption that the polymer molecules are much larger than the solvent molecules, the activity of the solvent $a_{\mathrm{s}}(-)$, can be described in terms of its volume fraction $\Phi_{\mathrm{s}}(-)$, and the volume fraction of the polymer $\Phi_{\mathrm{p}}(-)$ :

$$
\ln a_{\mathrm{s}}=\ln \Phi_{\mathrm{s}}+\Phi_{\mathrm{p}}+\chi \Phi_{\mathrm{p}}^{2}
$$

in which $\chi(-)$ is the polymer-solvent interaction parameter. Equation 4 shows that the activity of a solvent inside a polymeric layer is directly related to its volume fraction inside the swollen layer. Therefore, when modelling transport through polymeric membrane layers based on the solvent activity, it is important to know the solvent volume fraction inside the layer.

To account for the elastic contributions of a cross-linker, Equation 4 needs to be expanded with an contribution of the elastic deformation according to the Flory-Rehner theory [38]. The Flory-Rehner theory for swelling in $N$ dimensions is given in the Supplementary Information.

\subsubsection{Swelling of glassy polymers}

The swelling behavior of glassy polymers can be distinctly different from that of rubbery materials. Due to the presence of an excess free volume in glassy polymers, solvent uptake can occur both by polymer dilation and filling of the free volume (the so-called dual-mode sorption mechanism) [39,40]. The solvent uptake can in turn induce further polymer chain relaxations, which can occur over a broad range of timescales (seconds to years). Since glassy polymers show this non-equilibrium character, the Flory-Huggins theory does not hold for solvent uptake in glassy polymers [12,41-46]. Various attempts have been made to describe penetrant sorption in glassy systems mathematically [47-52]. However, the superposition of dilation, free-volume filling and secondary relaxations complicates the design of a unified 
theory. Therefore, in addition, semi-empirical relationships incorporating diffusional swelling and relaxations are being used to fit swelling data [53]. Because of the difficulty in modelling the swelling of glassy polymers, experimental data is essential for these systems.

Sporadically, solvent effects can even lead to a solvent-induced glass transition $[6,25,54-56]$

- an isothermal transformation of a glassy system into a rubbery system. Passing through the solvent-induced glass transition can lead to strong changes in the membrane properties. Moreover, for thin membrane films, the still poorly understood finite-size effects (i.e., nanoconfinement) can become very important, especially in the case of ultra-thin glassy films $[16,18,19,24,57]$. Such effects are minimized in this study by using films with thicknesses larger than $100 \mathrm{~nm}$.

\subsection{Models of polymer swelling dynamics}

The rate of solvent uptake by a polymer can be limited by two different processes: the diffusion rate of the solvent in the polymer and the relaxation of the polymer chains. These relaxations are a direct result of the solvent-induced swelling of the material. These two processes can be superimposed and therefore lead to multiple theoretical swelling curves.

\subsubsection{Rubbery polymers}

For rubbery polymers, the swelling by solvent uptake occurs almost instantly due to the absence of long-term polymer relaxations. Because the characteristic time scale for polymer chain mobility is of the same order of magnitude as the time scale for liquid diffusion, rubbery polymers usually attain equilibrium with the solvent very quickly (for thin supported films well below 1 second). Figure 2 displays the typical shape of the swelling-time and the concentration-time curve for the swelling of a (slightly cross-linked) rubbery polymer $(\mathbf{R})$. Upon solvent exposure, $t_{0}$, the thickness and normalized refractive index instantaneously attains their equilibrium values. 


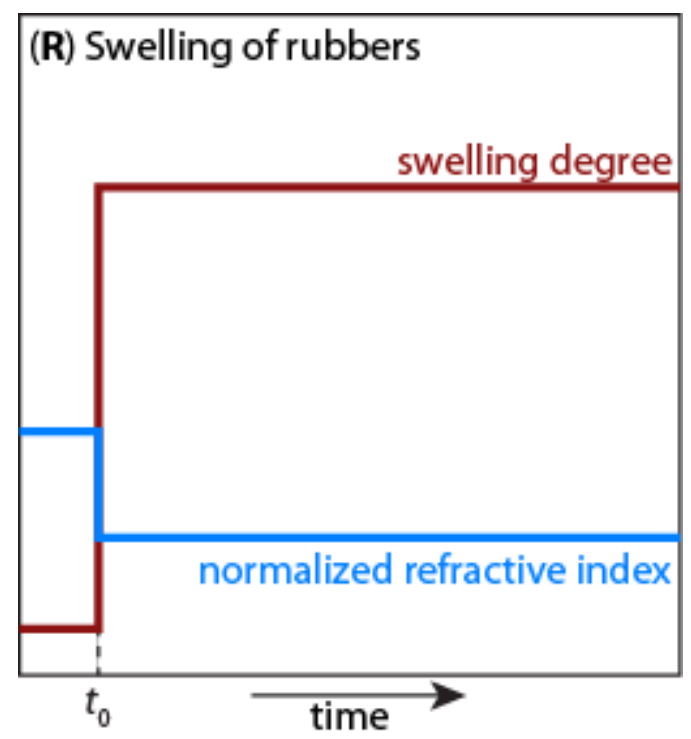

Figure 2: Master curve for the swelling of rubbers in a solvent, indicating the changes in the swelling degree and the normalized refractive index of the swollen layer. $t_{0}$ is the time of solvent exposure

The solvent that mixes with the polymer causes the refractive index $n_{\text {swollen }}$ of the swollen polymer to approach the refractive index of the solvent. Since rubbery materials do not contain excess free volume, the changes in thickness and refractive index are directly coupled. The refractive index of the swollen layer is a function of the refractive indices of polymer and solvent and their respective volume fractions $\left(\Phi_{\mathrm{s}}\right)$ in the layer, and can be expressed by an Effective Medium Approximation (EMA):

$$
\Phi_{\mathrm{s}} \frac{n_{\mathrm{s}}^{2}-n_{\text {swollen }}^{2}}{n_{\mathrm{s}}^{2}+2 n_{\text {swollen }}^{2}}+\left(1-\Phi_{\mathrm{s}}\right) \frac{n_{\mathrm{p}}^{2}-n_{\text {swollen }}^{2}}{n_{\mathrm{p}}^{2}+2 n_{\text {swollen }}^{2}}=0
$$

Hence, the volume fraction of the solvent inside the layer can be calculated based on the refractive indices of the solvent, the dry polymer and the swollen layer. It is equal to the solvent volume fraction calculation from the layer dilation, given in Equation 3.

\subsubsection{Glassy polymers}

Glassy polymers contain an excess free volume [58]. Due to this excess free volume, the changes in the thickness and the refractive index of a swollen glassy polymer are not fully coupled. For instance, a solvent could fill the free volume of the polymer, without causing significant changes in the thickness of the layer. As a result of this, at least four different types of swelling dynamics can be found when a glassy polymer is exposed to a solvent. An overview of these four cases is given in Figure 3. 

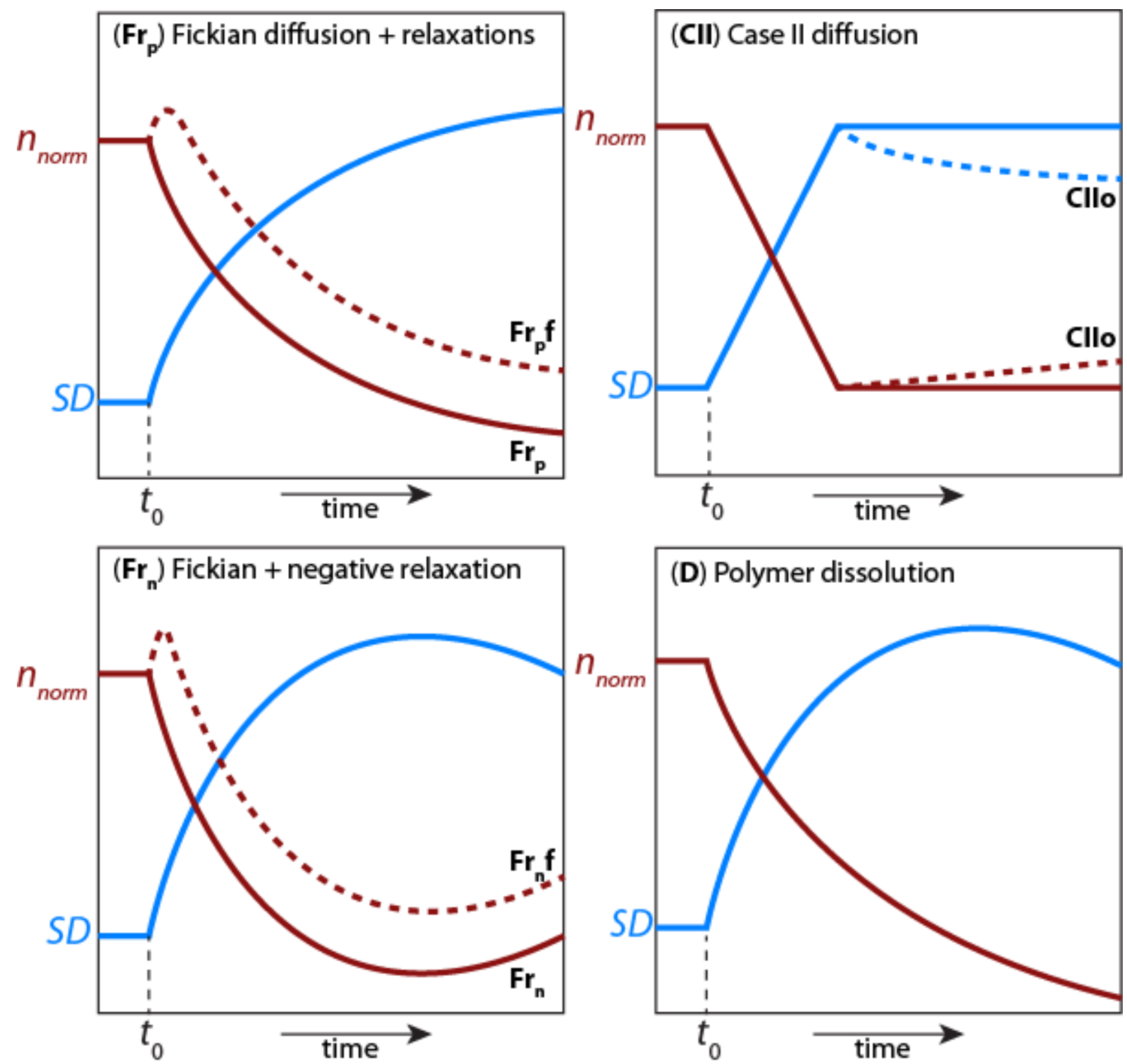

Figure 3: Master curves for the swelling of glassy polymers in a solvent, indicating the changes in the layer thickness and the layer's refractive index. $t_{0}$ is the time of solvent exposure. (top left) Fickian diffusion + positive relaxations, the dashed line indicates the special case where free volume filling takes place; (top right) Case II diffusion, the dashed line indicates the special case of overshoot dynamics; (bottom left) Fickian diffusion + negative relaxations, the dashed line indicates the special case where free volume filling takes place; (bottom right) Dissolution of the polymer into the solvent. Eventually, $S D$ will drop below zero.

Fickian Diffusion For most solvent-polymer combinations, the rate of dilation is governed by the Fickian diffusion (F) of the solvent through the polymer film. When plotted versus the square root of time, the swelling degree will initially show a linear behavior after which it will level off to the equilibrium swelling level. However, in many cases, this diffusion step is superimposed by secondary relaxations (r) of the polymer chains that can result in either a further dilation (positive relaxation, $\mathrm{rp}$ ) or shrinkage of the layer (negative dilation, $\mathbf{r}_{\mathrm{n}}$ ). 
In a polymer with a low excess free volume, the change in the refractive index reflects the change in the thickness closely. If the polymer, however, contains a significant amount of free volume (typical for polymers with a high $T_{\mathrm{g}}$ ), the free volume can be filled (f) by the solvent without showing a corresponding dilation; in this case, an initial increase in the refractive index of the swollen layer (since solvent replaces voids) is recorded.

Case II diffusion If the transport of the solvent is not limited by the Fickian diffusion, but only by the polymer relaxation, the so-called Case II diffusion (CII) occurs. Case II diffusion is characterized by a linear dependence of the dilation on time (note: in the manuscript, the experimental data is plotted versus the square-root of time for visualization purposes, and hence Case II diffusion will not display a straight line). Although a free-volume filling step (f) can take place, the changes during Case-II diffusion are typically too fast for recording the free volume filling within the temporal resolution of the experiment. Subsequent to Case II diffusion, the polymer typically shows so-called overshoot dynamics (o). Overshoot dynamics are essentially the same as positive relaxations, but were named differently in the case of Case II diffusion.

Dissolution If a solvent has a particularly high affinity for a (non-crosslinked) polymer, dissolution (D) of the polymer can occur. Typically, two type of dissolution models are discerned, of which normal dissolution is the most commonly applicable [59]. For normal dissolution, as shown in the bottom right panel of Figure 3, solvent diffuses into the layer, plasticizes the polymer into a gel layer that has rubbery characteristics, from which layer the polymer chains can disentangle and dissolve. Such behavior would be recorded by spectroscopic ellipsometry as a decreasing thickness while the refractive index stays constant or decreases as well. The thickness eventually decreases down to 0 , resulting in a swelling degree of -1. Alternatively, for thin films it is possible that the layer swells more strongly before disentanglement commences and no gel layer is formed. This phenomenon, which was not recorded in the pure solvents in our study, would be displayed by a continues swelling with simultaneous, continuous decrease in the refractive index, until at a certain point the refractive index of the polymer approaches the refractive index of the layer and the swelling approaches an asymptote. Opposed to the solvent 'eating away' layers from the top in the normal dissolution, the film swells until it is indistinguishable from the solvent and dissolves 'from within'.

\section{Materials and methods}




\subsection{Materials}

Matrimid $^{\circledR} 5218$ US (Huntsman), N-PAN (Dolan), PDMS (resin + crosslinker, Permacol ${ }^{\circledR}$ RTV 615, mixed in 10:1 ratio, Permacol ${ }^{\circledR}$ BV), PEBAX ${ }^{\circledR} 3533$ P2016 (Elf Atochem), PEI Ultem $^{\mathrm{TM}} 1000$ (GE Plastics), PES Ultrason ${ }^{\circledR}$ E6020P (BASF), PSf Udel ${ }^{\circledR}$ P-3500 (Amoco), PTMSP (Gelest Inc.), and P84 ${ }^{\circledR} 325$ mesh (HP Polymer) were used as received.

Table 1: Structural formulas of the polymers used for the swelling experiments.

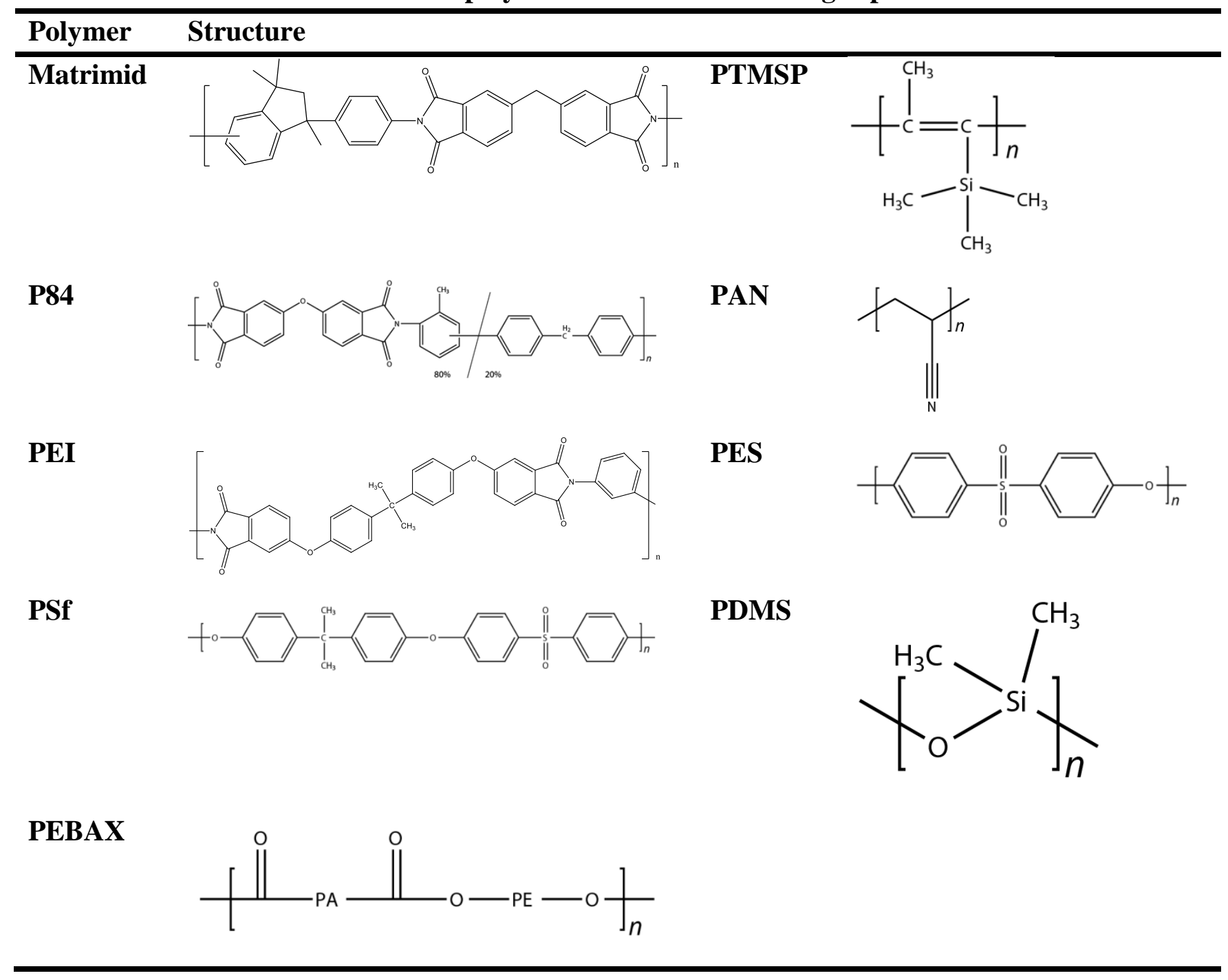

Cyclohexanone (Sigma-Aldrich, ACS reagent, $\geq 99.0 \%$ ), 1-butanol (Sigma-Aldrich, ACS Reagent, $\geq 99.4 \%$ ), NMP (Acros Organics, 99\%, extra pure), and toluene (99.9\% pure, EMSURE $^{\circledR}$, ACS, ISO, Reag. Ph Eur; Merck) were used as received for dissolving the polymers.

Silicon wafers (100, front side polished, CZ test grade, Silchem) were used as a substrate for the spin-coated layer. The wafers were stored under clean-room conditions until being cut. 
Prior to use, wafers were cleaned with acetone EMSURE $^{\circledR}$, ACS, ISO, Reag. Ph Eur; Merck).

Acetone (Chromasolv® plus, for HPLC 99.9\%, Sigma-Aldrich), 2-butanone (ACS reagent, $\geq$ 99.0\%, Sigma-Aldrich), ethanol (EMSURE ${ }^{\circledR}$, ACS, ISO, Reag. Ph Eur; Merck), ethyl acetate (p.a., ACS, ISO, Reag. Ph Eur, Merck), hexane (anhydrous, 95\%, Sigma-Aldrich), methanol (anhydrous, 99.8\%, Sigma-Aldrich), 2-propanol (LC-MS Chromasolv® $\geq 99.9 \%$, Fluka or anhydrous, $>99.5 \%$, Sigma-Aldrich), toluene (anhydrous, $\geq 99.8 \%$, Sigma-Aldrich), and dichloromethane (Sigma-Aldrich) were used as received for the swelling study.

Nitrogen used for the inert atmosphere during the $T_{\mathrm{g}}$ and EFV analysis was purified using a

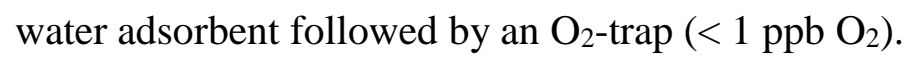

\subsection{Layer preparation}

Thin polymer films were prepared by spin coating (WS-400B-6NPP/LITE, Laurell Technologies Corporation) of a polymer dope onto a silicon wafer under a nitrogen atmosphere. Polymer dopes were prepared with the solvents and concentrations listed in Table 2. Silicon wafers cut to pieces of approximately $2 \times 2 \mathrm{~cm}$. Prior to spin coating, wafers were cleaned with acetone, wiped with a paper towel and let to dry. The spinning dope is applied as soon after drying as possible to prevent contamination of the surface. After applying the spinning dope, a wait time of $\sim 30$ seconds was used to equilibrate the temperature of the dope and the wafer. The general spin coating recipe consisted of a single step at a speed of 3000 for 60 seconds. For polymers dissolved in NMP, an alternative recipe was used, which consisted of a 10 seconds step at $500 \mathrm{rpm}$, followed by a 2.5 minute step at $2500 \mathrm{rpm}$, and a 4 minute drying step at $625 \mathrm{rpm}$ to evaporate the NMP from the sample. The thickness was tuned by changing the concentration of the spinning dope (see Table 2), in order to obtain layers with dry thicknesses of 100-300 nanometer.

The excess free volume (EFV) and glass transition temperature $\left(T_{\mathrm{g}}\right)$ were measured using temperature-dependent spectroscopic ellipsometry analysis (TEA) on an M2000-X ellipsometer (J.A. Woollam Co.) equipped with a temperature-controlled hot-stage (HCS622, INSTEC), the temperature was calibrated using melting point standards [60]. The spectroscopic ellipsometry measurements were conducted in the full wavelength range of $370-1000 \mathrm{~nm}$. 
During the experiments, the hot stage was continuously purged with ultrapure nitrogen. Prior to the thermal treatment, the films were kept at room temperature for 30 minutes. Subsequently, the samples were heated instantaneously to a temperature well above the $T_{\mathrm{g}}$ but well below the degradation temperature for 2 hours. The sample was held at this temperature for 2 hours and subsequently cooled down with a cooling rate of $5{ }^{\circ} \mathrm{C}$ per minute. This procedure was repeated multiple times, to find the highest possible temperature at which degradation did not occur and to obtain data for statistical error analysis. To obtain the hypothetical liquid refractive index, the procedure outlined in reference [36] was followed.

Data analysis was performed with the same models that were used for the dry layers. For the silicon wafer, the temperature-dependent dielectric function provided by CompleteEASE was used.

Table 2: Polymers, solvents, concentrations used in preparation of the thin films, their average refractive index, excess free volume and $T_{\mathrm{g}}$. Error bars indicate the standard error.

\begin{tabular}{llccccc}
\hline Polymer & Solvent & $\begin{array}{c}\text { Concentration } \\
(\text { wt- } \%)\end{array}$ & $\begin{array}{c}\text { Annealing } \\
\text { temperature } \\
\left({ }^{\circ} \mathbf{C}\right)\end{array}$ & $\begin{array}{c}\text { Refractive index } \\
\text { at 632.8 nm }\end{array}$ & EFV & $T_{\mathbf{g}}\left({ }^{\circ} \mathbf{C}\right)$ \\
\hline Matrimid & Cyclohexanone & 4.8 & 235 & $1.6393 \pm 0.0008$ & $15.8 \pm 0.2 \%$ & $337 \pm 9$ \\
P84 & NMP & $7.5-10$ & 335 & $1.6795 \pm 0.0002$ & $11.9 \pm 1.2 \%$ & $346 \pm 7$ \\
PES & NMP & $7.5-10$ & 230 & $1.6527 \pm 0.0009$ & $7.1 \pm 1.0 \%$ & $232 \pm 3$ \\
PSf & NMP & $7.5-10$ & 205 & $1.6296 \pm 0.0002$ & $7.3 \pm 0.5 \%$ & $197 \pm 3$ \\
PEBAX & Butanol, reflux at 60 & 4 & 80 & $1.4867 \pm 0.0004$ & & \\
& ${ }^{\circ}$ C, spin-coating at & & & & & \\
PTMSP & Toom temperature & & & & & \\
PAN & NMP & 3 & 250 & $1.4868 \pm 0.0007$ & & \\
PEI & NMP & $7.5-10$ & 110 & $1.5153 \pm 0.0003$ & $1.4 \pm 0.1 \%$ & $95 \pm 2$ \\
PDMS & Toluene & $5.5-10$ & 235 & $1.6580 \pm 0.0004$ & $8.0 \pm 0.3 \%$ & $216 \pm 1$ \\
\hline
\end{tabular}

For the rubbery polymers PDMS and PEBAX, the $T_{\mathrm{g}}$ is below room temperature and no EFV is present at room temperature. PTMSP could not be annealed above the $T_{\mathrm{g}}$ without degradation and as such, the $T_{\mathrm{g}}$ and EFV could not be determined.

In order to obtain a representative starting point for the glassy polymers, the polymers were annealed for 8 hours (see Table 2). All polymers except for Matrimid, PEBAX, PTMSP, and PDMS were annealed above their $T_{\mathrm{g}}$, To minimize aging the effects of aging that could occur after the annealing, all polymers were stored under nitrogen for exactly 6 days prior to use. 


\subsection{Measurement protocol}

For all polymer-solvent combinations, the following measurements were performed (after the annealing and 6 days ageing period):

1. Dry thickness at 9 random spots on the sample;

2. The thickness in the solvent at a single spot continuously for at least 8 hours;

3. Swollen thickness in the solvent at 9 random spots after the continuous measurement;

4. Swollen thickness in the solvent at 9 random spots after two weeks;

5. Swollen thickness in the solvent at 9 random spots after four weeks;

6. Swollen thickness in the solvent at 9 random spots after six weeks or longer;

7. Dry thickness at 9 random spots after fully drying of the polymer layer.

In the long-term data, the data points are connected by lines. These lines form a guide-to-theeye only.

\subsection{In situ measurement of swelling: layer thickness and solvent concentration}

The layer thickness and refractive index were determined from $(\Psi, \Delta)$-spectra, obtained using spectroscopic ellipsometry on a M-2000X or $\alpha$-SE ellipsometer (both J.A. Woollam Co., Inc.) under an angle of incidence $70^{\circ}$. For all except the short-term measurement, multiple spots $i$ are measured on a sample to get information on the sample inhomogeneity that can be used in the analysis of the experimental uncertainty. Measurements in solvents were performed in a temperature-controlled glass cell at $22{ }^{\circ} \mathrm{C}$. The unavoidable time lag of approximately 10-30 seconds between inserting the polymer in the solvent and the first measurement point by the ellipsometer was recorded and corrected for in the time data.

\subsubsection{Data modelling}

Here, a summary of the data modelling procedure is given. The full details on the modelling are given in the Supplementary Information. All films were modelled using a silicon wafer [61] with $2 \mathrm{~nm}$ native oxide [61] as a substrate. Where possible, the polymer films were modelled by a simple, isotropic Cauchy model. For some polymers, more advanced models were required to capture the data successfully. For instance, a gradient in the refractive index, or an optical anisotropy in the film had to be taken into account. These advanced cases are indicated by an asterisk (*) in the manuscript, and are explained in full detail in the Supplementary Information. For the polymers exposed to the solvent, the optical dispersion of the solvent was included. 
For the purpose of visualisation, the dynamic data are plot versus the square root of time; this representation does not imply any physical mechanism.

\subsection{Quantification of experimental uncertainties}

Uncertainties on obtained values are inherent to experiments. In the case of measuring swollen films using spectroscopic ellipsometry, measurement inaccuracies can be introduced easily. The technique performs optimally on smooth and laterally homogeneous films in the range of 100-500 nanometer, which fortuitously is the range of interest for coated membrane films. Since spectroscopic ellipsometry relies on an optical contrast between a (swollen) layer and its environment (the solvent), it is especially sensitive to perturbations when the refractive index of the polymer film approaches the refractive index of the solvent, which is the case for highly swollen films (and in some cases, already for the dry polymer-solvent combination). Readers unfamiliar with spectroscopic ellipsometry are referred to Ogieglo et al. [30] for a clear description of the advantages, disadvantages and limitations of using spectroscopic ellipsometry for measuring swelling polymer films. For a thorough discussion on possible errors and inherent measurement uncertainties, the reader is referred to Tempelman et al. [62]. Throughout this manuscript, uncertainties are quantified and given as error bars, which denote the standard error. For those data points for which uncertainties in the data value are introduced both through the data fit and through the lateral inhomogeneity of the sample, the error bar gives the root mean square of both errors.

\section{Results and discussion}

\subsection{PDMS}

PDMS (polydimethylsiloxane) is a popular material in, amongst other applications, membranes [1] and microfluidics [63]. It is a rubbery polymer, with one of the lowest known glass transition temperatures $\left(-120^{\circ} \mathrm{C}\right)$. PDMS is inert, optically transparent, non-toxic, nonflammable and is easily molded into many forms or coated onto a substrate. In membrane technology, PDMS has initially been applied by Monsanto as a repair coating on PSf gasseparation membranes [64]. In organic solvent nanofiltration, early work on PDMS was performed at major oil and chemical companies (see, e.g., [65]). PDMS still is a material of choice for many membrane applications, such as organic solvent nanofiltration, pervaporation, and gas separation. The swelling behavior of PDMS in organic solvents is well documented [66-71]. The results given in this section therefore serve to demonstrate the consistency between swelling data obtained using spectroscopic ellipsometry in this study and 
swelling data obtained using mass-based solvent uptake measurements on (semi)bulk samples [71] and bulk dilation [66].

\section{Long-term swelling}

Figure 4 shows the long-term swelling behavior of PDMS samples over a measurement period of 2 months. The swelling is displayed in two ways. The left panel shows the swelling degree, which is the ratio between the swollen thickness and the thickness of the dry layer, the right panel displays the normalized refractive index, running from 1, which indicates a dry polymer ( $n_{\text {swollen }} \approx n_{\text {polymer }}$, to 0 , indicating that the swollen layer consists of nearly only solvent ( $n_{\text {swollen }} \approx n_{\text {solvent }}$ ) (see the definition of Equation 2 ). In the case of PDMS, both the swelling degree and the normalized refractive index stay constant over the complete measurement period of 2 months. The absence of changes on this time scale is consistent with the behaviour of an equilibrium polymer above its glass transition. Upon exposure to a solvent, an equilibrium is reached almost instantaneously between sample and solvent. Afterwards, the polymer swelling remains stable over at least the two-month timespan of our measurements.

In the solvents in which PDMS shows the highest swelling degrees, the dilation is only restricted by the cross-linking of the polymer (cf. [32]), without which the layers would have dissolved. Listed from largest to smallest, the order of solvent swelling is: apolar solvents (hexane, toluene) > polar aprotic (EA, MEK, acetone) > polar protic (IPA, ethanol, methanol, water). This order is consistent with measurements of the solvent mass uptake and bulk dilation of PDMS in previous studies (cf. Supplementary information, Figure S1) $[66,71]$.

The changes in the normalized refractive index of the layers correspond to the changes in the swelling. As expected for a rubbery polymer, the normalized refractive index is low for highly swollen layers and vice versa. The order of decrease in the normalized refractive index corresponds to the order of increase in swelling degree. As the observed swelling degrees for PDMS reaches of well above $100 \%$, the optical contrast between the swollen PDMS layer and the surrounding solvent diminishes. This effect is even stronger for solvents with a refractive index very close to that of PDMS (i.e., MEK, IPA, EA, and hexane). This results in an increased experimental uncertainty compared to the other polymers in both the swelling degree and $n_{\text {normalized }}$ compared to other polymers. 

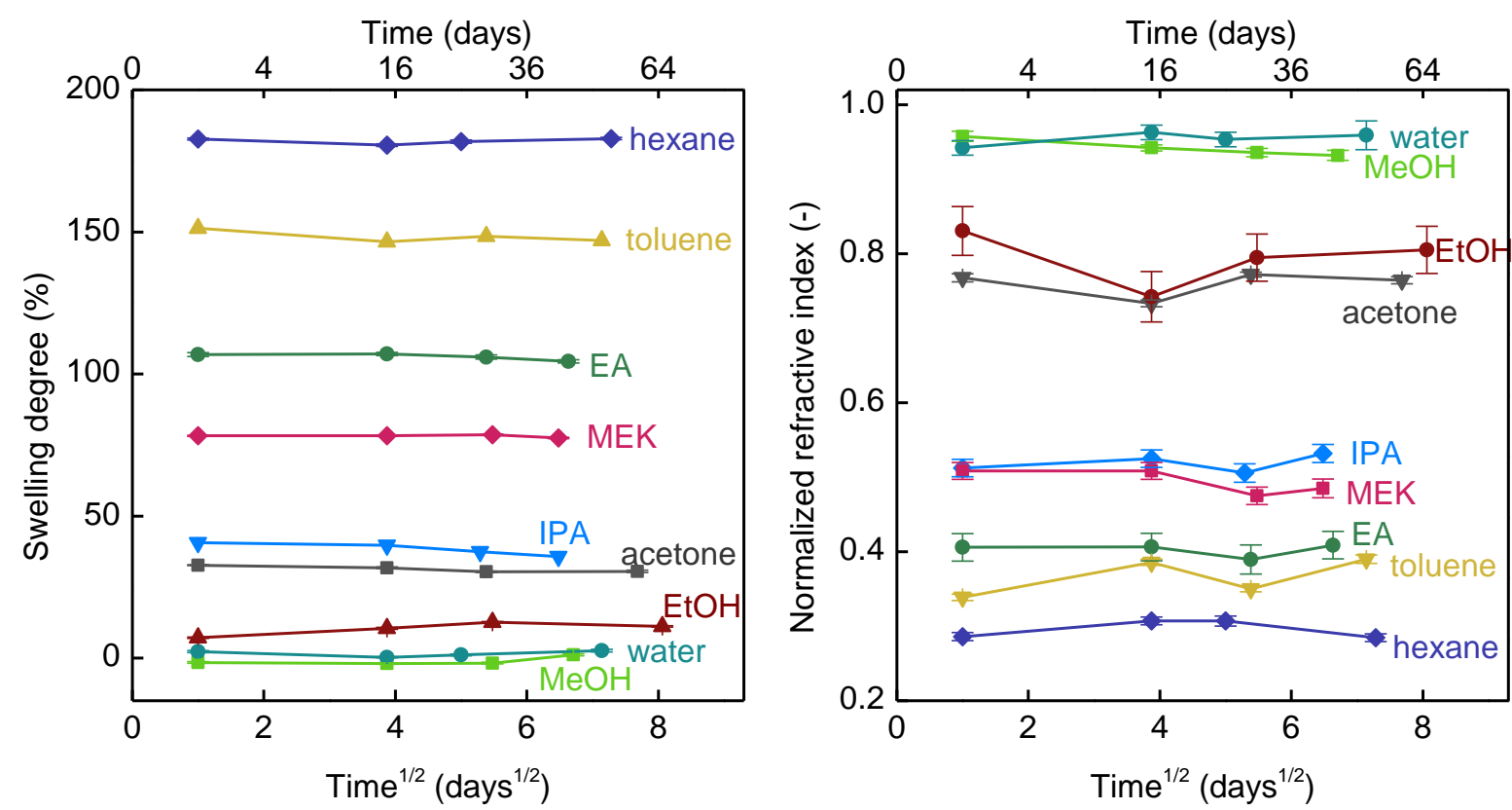

Figure 4: Swelling degree (left panel) and normalized refractive index (right panel) of PDMS as a function of time in the solvent. The swelling of PDMS in DCM could not be measured due to similar refractive indices: $n_{\text {PDMS }}(1.41) \approx n_{\text {DCM }}(1.42)$.

For solvent uptake by rubbery materials, volume additivity of the polymer and solvent should hold, in accordance with Henry's law. The solvent fraction can be calculated independently from the dilation using Equation 3 or from the refractive index of the swollen layer by Equation 5. Volume additivity is revealed from the fact that the solvent volume fractions calculated from the swelling data $\left(\Phi_{\mathrm{s}, \mathrm{swelling}}\right)$ and calculated from the refractive index data

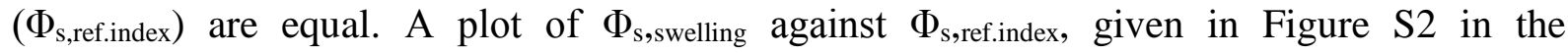
Supplementary Information, shows a slope parallel to the $x, y$-line, with a small offset. This offset can originate from non-ideal mixing behavior [12] or from experimental uncertainties. Since the dilation and refractive index are obtained independently (i.e., with minor covariance in the ellipsometry data fit), the consistency of the two calculations within the experimental errors demonstrates the high accuracy of the ellipsometry measurements in determining both variables.

In addition to recording the swelling over longer times, we also investigated the swelling dynamics in situ during the first 10 hours of swelling. By tracking a single measurement point over the course of 10 hours, an accurate measurement of the swelling dynamics of the polymer films was obtained. However, compared to the long-term measurements where averaging over multiple measurement points with respect to the dry thickness was possible, the precision of the in situ swelling measurement is inherently lower (see Section 3.5). 


\section{Short-term dynamics}

Figure 5 shows the swelling degree (left panel) and normalized refractive index (right panel) of PDMS films during the first 10 hours following on solvent exposure. For both the swelling and normalized refractive index, the obtained values compare well with the long-term data, especially considering the uncertainty on the values of the normalized refractive index, which are due to the minor optical contrast between the solvent and the swollen layer.

For all solvents, the swelling degree instantly attains its equilibrium value, implying that the swelling takes place within the first seconds of the measurement. This behavior is typical for rubbery materials [72] and consistent with the rubbery swelling model (R).
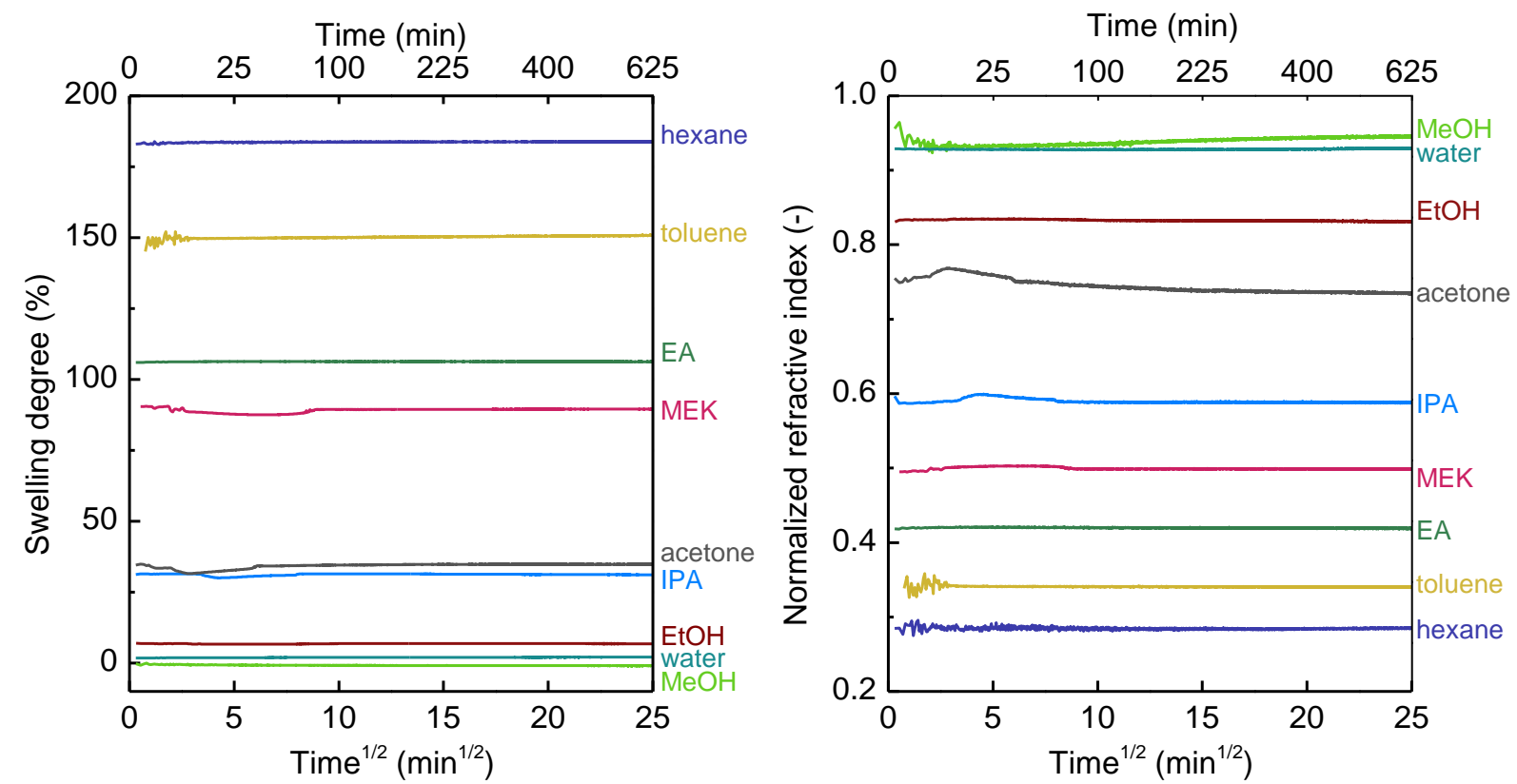

Figure 5: Swelling degree of PDMS as a function of time for the first 10 hours of swelling, given by the swelling degree (left panel) and normalized refractive index (right panel).

\subsection{Polyimides}

Polyimides (in this study P84, PEI and Matrimid) are mechanically stable and chemically robust glassy polymers with high glass transition temperatures. Their high excess free volume in combination with their glassy nature and temperature stability up to $200^{\circ} \mathrm{C}$, sparked the initial interest in polyimides for gas separation membranes [73,74]. In organic solvent nanofiltration, cross-linked polyimides find their application as supports or as selective layer [1]. The first major-scale OSN process (Max DeWax) used polyimide-based membranes [75].

\subsubsection{P84}


P84 (produced by Evonik, formerly Lenzing) is a high-performance engineering polymer used for high-temperature applications including gas separation and hot-gas filtration [76]. Since P84 has a high stability at elevated temperatures and in aggressive solvents, it was one of the first polymers to be applied as an OSN membrane at a major industrial scale as the STARMEM $^{\circledR}$-membrane in the Mobil Max DeWax-process [77]. P84-based membranes have been used in solvent recovery, solvent exchange and solute enrichment processes [78]. Note that the properties of the P84 layers measured in the present work may differ from those of Evonik membranes, as the latter membranes are made via proprietary production processes, involving a cross-linking reaction of the polymer network [79].

\section{Long-term swelling}

Figure 6 shows the long-term development of the swelling (left panel) and normalized refractive index (right panel) of P84 over a measurement period of 2 months. All solvents induce a swelling that progresses over a timescale of months. After 2 months of solvent exposure, the P84 films still display no sign of equilibration. In DCM, the swelling is most pronounced with a swelling degree of $30 \%$ after 2 months. The polar aprotic solvents acetone, MEK, and EA show significant but somewhat lower swelling levels as compared to DCM. The normalized refractive index roughly mirrors the swelling trends, slowly moving away from a dry polymer towards more solvent-like properties. In the other solvents (methanol, ethanol, isopropanol, water, hexane, and toluene), the swelling degree after two months is below $10 \%$ but still increasing. The normalized refractive indices of the swollen layers show trends corresponding to the trend of the swelling degree, although the measurement uncertainties introduce some scatter on the data. Except for hexane, the data show a decreasing normalized refractive index, a trend that is consistent with a continuous swelling of the layer. The normalized refractive index is higher than may be expected based on the swelling level, which can be explained by the replacement of free volume void space by solvent. Since the normalized refractive index is smaller than 1, effects of dilation on the refractive index value of the polymer outweigh the effects of void filling. 

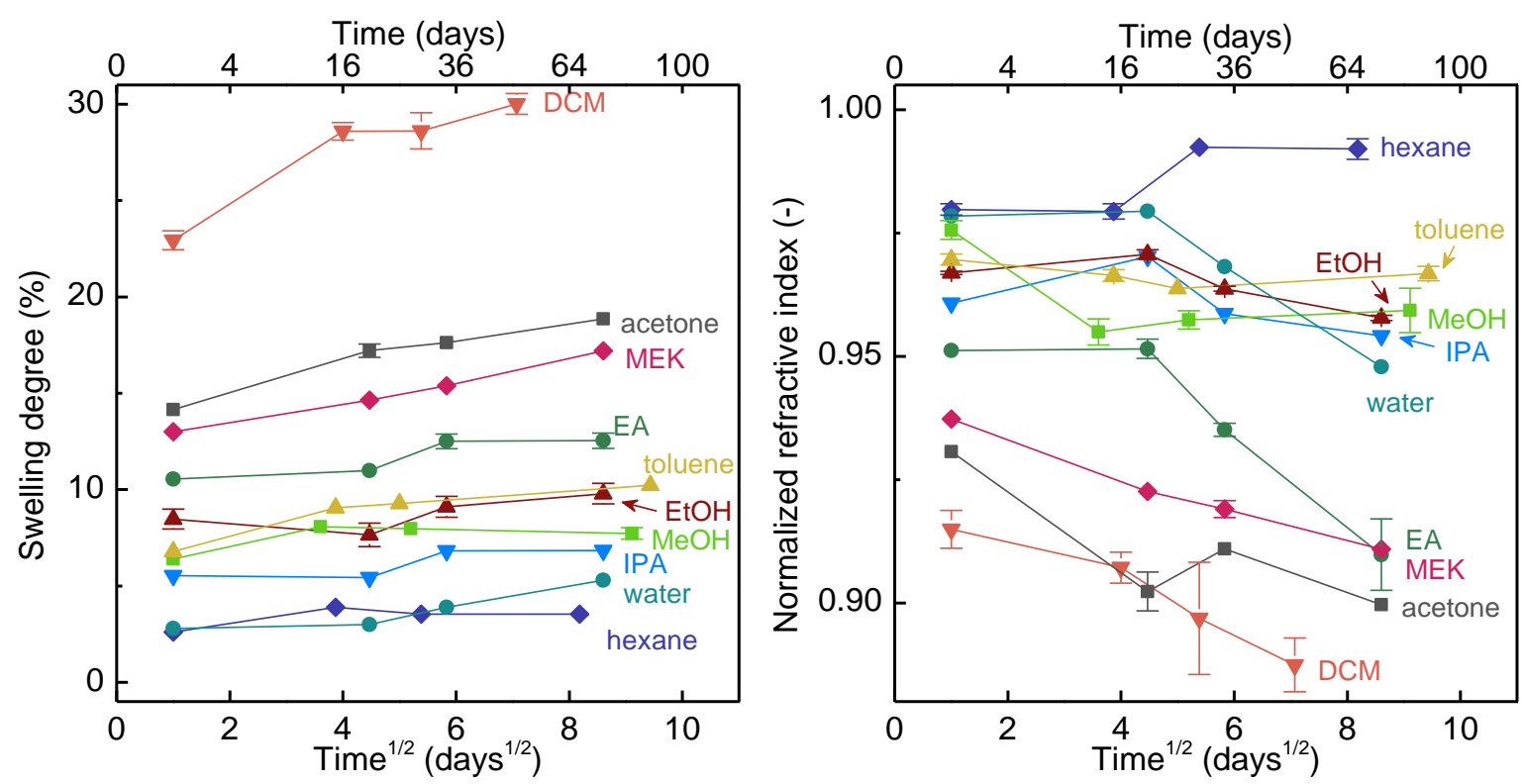

Figure 6: Swelling degree (left panel) and normalized refractive index (right panel) of P84 as a function of time in the solvent.

\section{Short-term dynamics}

Figure 7 shows the swelling (left panel) and normalized refractive index (right panel) of P84 layers during the first 10 hours after solvent exposure. As for the long-term data, for all solvents the measured swelling of P84 within the measured timeframe is not an equilibrium value but a kinetically limited swelling value.

For P84, the swelling mechanisms cannot be categorized according to the solvent type (apolar, polar protic and polar aprotic). In DCM, acetone, methanol, ethanol, and hexane, the swelling follows the Fickian-diffusion followed by positive relaxations mechanism $\left(\mathbf{F r}_{\mathbf{p}}\right)$, which is manifested through a strong initial swelling followed by a slowly progressing swelling over very long timescales. Here, after an initial swelling step, the swelling appears to increase approximately linearly with the square root of time.

In the other solvents, MEK, EA, IPA, and toluene, the picture is more complex. The isotropic Cauchy model proved inadequate to model the refractive index and thickness of swollen P84 layer accurately. Particularly during the first minutes following the solvent exposure, the isotropic model resulted in a high mean square error. Therefore, we have evaluated multiple optical models that are capable of capturing non-isotropic behavior. The full details of this evaluation are given in the Supplementary Information. The best and most realistic fits were obtained by a model that includes a gradient in the refractive index perpendicular to the substrate. The use of this model is indicated by the asterisk in the figure. 
A gradient in the refractive index is essentially a gradient in the density of the layer. Physically, this gradient can be caused by a graded solvent concentration inside the layer. Such a gradient forms when the solvent uptake of the layer is diffusion limited. As the solvent front progresses through the polymer layer over time, the gradient disappears. The optical model confirms the disappearing density/solvent gradient in our data over time, up to the point where the gradient is completely absent. For this reason, the long-term data in Figure 6 could be modelled with the isotropic model.

For most of the solvents, an offset in the refractive index at the start of the measurement is recorded. This offset can be the consequence of an inaccuracy that is either caused by the introduction of the solvent into the measurement cell as shown by Tempelman et al. in [62], or is the consequence of an initial process that is too fast (in the timescale of seconds) to capture using spectroscopic ellipsometry. Upon comparing the data at the end of the shortterm measurement to the more accurate data obtained on multiple sample spots after 1 day (Figure 6), it is seen that a small offset is still present at the end of the short-term data. This indicates that this offset is present over the whole data trend. Therefore, the short-term data should be used to investigate trends, whereas the long-term data provides accurate values for the swelling degree and (normalized) refractive index.

Both MEK and EA show a clear increase in the normalized refractive index during the first minutes following on solvent exposure. This increase is consistent with a filling of P84's free volume, indicating an Frpf-mechanism. A similar increase, albeit taking place slower, is seen for hexane, toluene, and IPA. For these layers, after an initial drop in the normalized refractive index, it does not decrease further, but even increases over time. This increasing normalized refractive index in combination with the dilation of the layer can only be rationalized by a free-volume filling effect. As such, the main swelling of the layer had to take place on the same time scale as the free volume filling, resulting in only minor relaxations at longer timescales. Whether the other solvents fill the free volume as well cannot be concluded from the data; the absence of an increase in the refractive index is possibly due to the freevolume filling effect being outweighed by the simultaneous swelling of the material. 

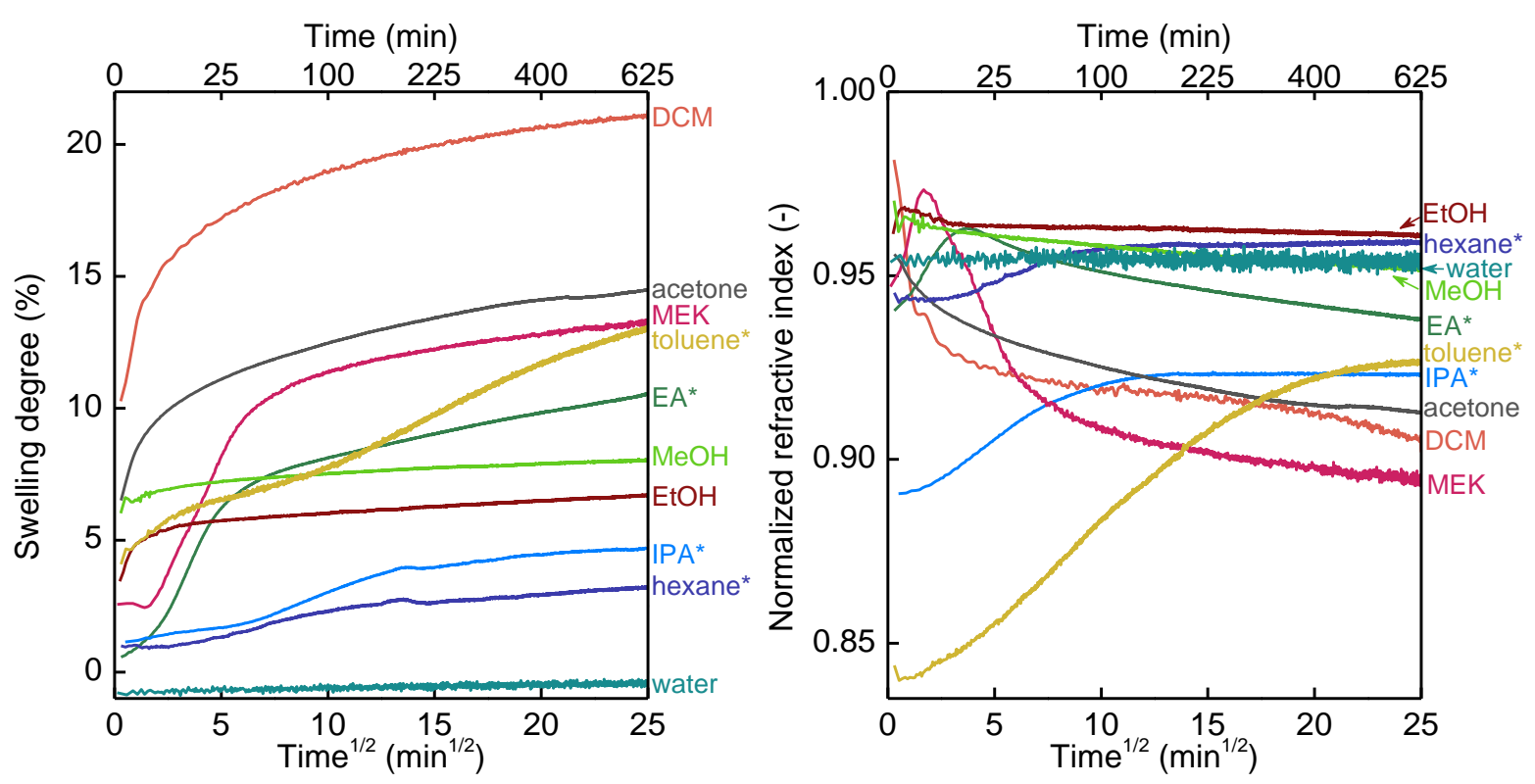

Figure 7: Swelling dynamics of P84 as a function of time for the first hours of swelling, given by the swelling degree (left panel) and the normalized refractive index (right panel). The asterisk indicates the use of a gradient model (see Supplementary Information). The data for DCM was smoothed (untreated data in the Supplementary Information, Figure S14)

\section{Final remarks on P84}

Our ellipsometry-based measurements of the swelling degree are consistently higher than the values that have been previously obtained in other studies using the solvent mass-uptake method: in most solvents, the swelling degree measured in this study is far higher than the previously recorded solvent mass uptake. Table 3 gives a comparison of the data obtained in our study with data obtained in other studies $[80,81]$. The difference in swelling degrees between our data and the literature data cannot be explained by measuring the swelling in 3 dimensions (mass uptake) vs. 1 dimension (ellipsometry) (see a discussion on conversion of the swelling degree between 1D and 3D in the Supplementary Information). What could cause the difference is the fact that in thicker samples, gradients will persist over much longer times. Additionally, differences can occur through measurement inaccuracies in the mass-uptake method or differences in the polymer batches (for instance, its molecular weight, molecular weight distribution, or changes in the polymer's synthesis recipe by the manufacturer). 
Table 3: Comparison of swelling degree of P84 (this study) with swelling degrees obtained via the mass uptake method [80,81]

\begin{tabular}{lccc}
\hline Solvent & $\begin{array}{c}\text { Swelling degree } \\
(\sim 2 \text { months) } \\
\text { (this study) }\end{array}$ & $\begin{array}{c}\text { Mass uptake } \\
(\mathbf{1 0 0 - 6 0 0} \text { days }) \\
{[80]}\end{array}$ & $\begin{array}{c}\text { Mass uptake } \\
(\mathbf{1} / 3 \text { months }) \\
{[81]}\end{array}$ \\
\hline Toluene & 10.2 & 2.7 & \\
EA & 12.5 & 2.8 & \\
Hexane & 3.5 & 1.4 & \\
Ethanol & 9.8 & 8 & $1.7 / 7.4$ \\
IPA & 6.8 & $<1$ & $0.6 / 2.3$ \\
Water & 5.3 & 5.8 & $3.5 / 6.8$ \\
\hline
\end{tabular}

In summary, the long equilibration times recorded for the swelling of P84 are a result of the strong glassy character of the polymer. As a consequence, reliable characterization of the membrane performance of P84 in the presence of solvents can only be obtained through longterm measurements. Interpretation of data obtained during the first hours after solvent exposure may lead to false conclusions regarding the performance (flux and selectivity) of the polymer. In all solvents, the swelling of P84 follows a combination of Fickian diffusion of the solvent combined with positive relaxations of the polymer chains $\left(\mathbf{F r}_{\mathbf{p}}\right)$, in some cases combined with free-volume filling ( $\left.\mathbf{F r}_{\mathbf{p}} \mathbf{f}\right)$. On what timescale the swelling of the P84 films equilibrate and to which extend cross-linking of the film affects this process, remains a topic of further studies.

\subsubsection{PEI}

Structurally related to the other polyimides, PEI is a high- $T_{\mathrm{g}}$ polymer with a high mechanical strength. Processing of PEI via phase inversion is known to be tedious and often results in membranes with relatively low fluxes and low rejections [82]. Nevertheless, for some organic solvents PEI could provide an interesting alternative to the other polyimides, as it is cheaper.

\section{Long-term swelling}

Figure 8 shows the long-term development of the swelling (left panel) and normalized refractive index (right panel) of PEI over a measurement period of 2 months. Similar to P84, the swelling of PEI does not reach equilibrium within this period. In DCM, PEI dissolved (d) readily. In the polar aprotic ketones (acetone, MEK, and EA), the swelling degree is the highest, and gradually increases over the course of time, with a corresponding decrease in the normalized refractive index. PEI also swells significantly in toluene, in which strong changes are recorded over the course of the full two months. This continuous but slow swelling may be the result of a plasticization of the PEI by the increasing toluene content. As for P84, in 
hexane the swelling of PEI is minor. In alcohols, swelling degrees below 5\% are recorded after 1 day, which are slightly lower than the swelling degrees for P84 in the alcohols. After this initial swelling, further changes in the swelling are limited. Nearly no swelling was recorded in water. The normalized refractive indices of the layers that were swollen in the alcohols, hexane, toluene, and water show only minor changes in the order of magnitude of the error margin.
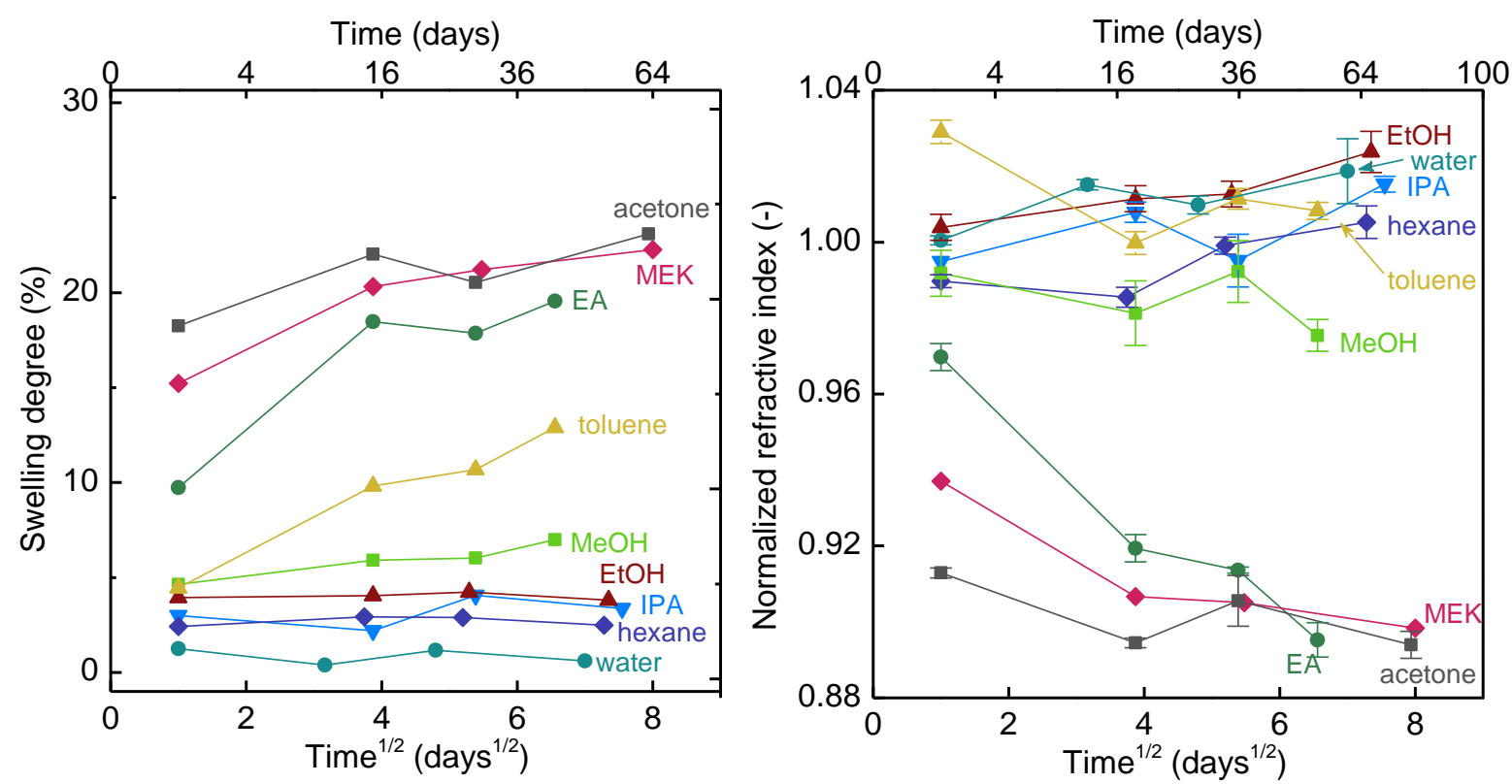

Figure 8: Swelling degree (left panel) and normalized refractive index (right panel) of PEI as a function of time in the solvent. PEI dissolved in DCM.

\section{Short-term dynamics}

Figure 9 shows the swelling (left panel) and normalized refractive index (right panel) of P84 layers during the first 10 hours after solvent exposure. The swelling degrees are consistent with the long-term data. For the layers that are swollen by the ketones, the swelling could not be modelled with a homogeneous layer. Instead, a model with a gradient in the refractive index (i.e., a density gradient) best captured the swelling of the material. The use of this model is indicated by an asterisk in Figure 9; the reader is referred to the Supplementary Information for full details. This density gradient can physically be interpreted as a distributed solvent concentration perpendicular to the substrate. The swelling dynamics of PEI in the ketones adhere to a Fickian diffusion model, followed by positive relaxations of the polymer chains. During the first minutes, the swelling is accompanied by the filling of the free volume in the PEI layer $\left(\mathbf{F r}_{\mathbf{p}} \mathbf{f}\right)$, which is apparent from the increase in the normalized index upon solvent exposure (for a discussion of the offset in $n_{\text {norm }}$ at $t=0$, see Section 4.2.1). 
Hexane, the alcohols, and water show minor swelling levels of 2-5\%, and correspondingly, there is only little change in the normalized refractive index. The swelling takes place following the Fickian-diffusion mechanism $(\mathbf{F})$. Relaxations of the polymer chains are very small or absent; even in the long-term data, nearly no change is recorded. The swelling of PEI in toluene and hexane are vastly different, even though both solvents are apolar. In toluene, at first only a minor swelling is recorded, followed by a plateau in the swelling degree after half an hour. At this point, the refractive index continuously changes, which can be indicative for the progressing filling of the excess free volume inside the material. After a few hours in the solvent, the swelling degree increases again, whereas the changes in the index of the layer reach a plateau.
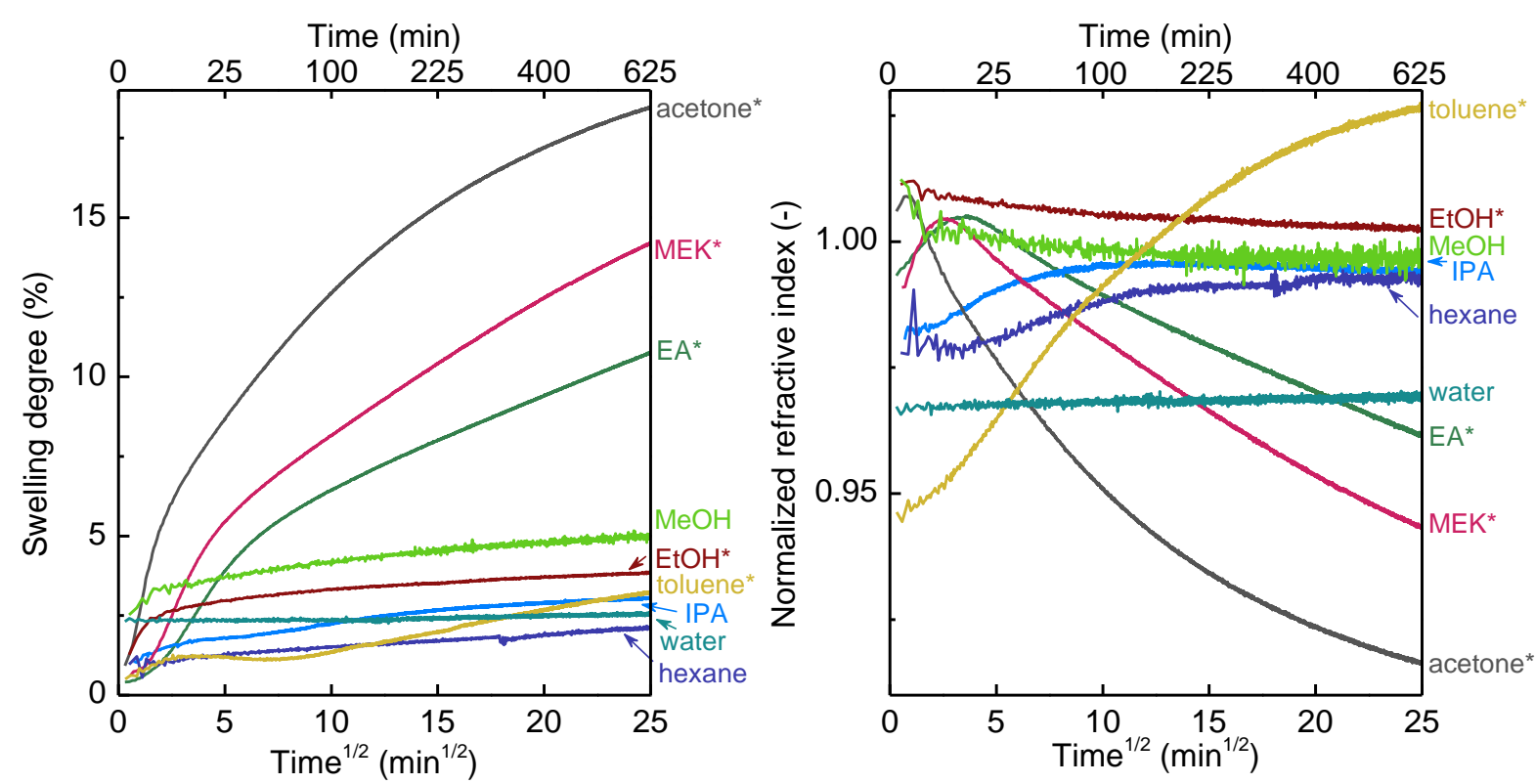

Figure 9: Swelling dynamics of PEI as a function of time for the first hours of swelling, given by the swelling degree (left panel) and the normalized refractive index (right panel). $\Delta S D /(S D+1)=0.05$ (toluene), $<0.02$ (all other solvents); $\Delta n_{\text {norm }}=0.14$ (toluene), 0.07 (hexane and ethanol), 0.06 (EA, IPA, MEK), and $<0.05$ (all other solvents).

\section{Final remarks on PEI}

Literature data on the swelling of pure PEI is limited. Using the mass uptake method, swelling degrees of $1.38 \mathrm{wt}-\%$ and $1.44 \mathrm{wt}-\%$ were measured for water and ethanol, respectively [83]. Those values deviate slightly from the dilation values found in this study (0.6 and 3.8\%, respectively). The difference is smaller than the inherent inaccuracy of the solvent massuptake technique and the error bars on our data [62].

The slow swelling rates and continuous changes reflect the glassy character of PEI. The similarity to P84 in swelling degree and swelling dynamics could imply that PEI is a 
potentially suitable OSN membrane layer, if a stabilization of the layer through cross-linking similar to that being employed for P84 can be achieved.

\subsubsection{Matrimid}

Most polyimides are hardly soluble after they have been imidized. In contrast, Matrimid is directly soluble in many polar aprotic solvents [84]. Inherently, the strong affinity to these solvents limits its application as an OSN membrane, even when the membrane is stabilized by a cross-linking procedure [1]. Therefore, and because of its high free volume, the main application of Matrimid is as a gas separation membrane.

Spin-coated layers of Matrimid can demonstrate structural anisotropy [85]. This anisotropy can manifest itself in birefringence (i.e., a different refractive index, depending on the polarization of incoming light). As a result of this, modelling the layer as an isotropic film failed and a model that incorporates optical anisotropy has been used for analyzing Matrimid data. When the layers swell significantly, the polymer relaxes and the anisotropy disappears. A comparison of the models is provided in the Supplementary Information.

\section{Long-term swelling}

Figure 10 shows the long-term development of the swelling (left panel) and normalized refractive index (right panel) of Matrimid over a measurement period of 2 months. In DCM, Matrimid dissolved (D). The strongest swelling is recorded for toluene, in which Matrimid shows a swelling degree of $70 \%$ after one day, after which changes over longer time scales are virtually absent. For the three polar aprotic ketones (MEK, acetone, and EA), swelling degrees of $50-60 \%$ are reached. Whether further changes through positive or negative relaxations take place cannot be concluded, since the changes in the data are within the margin of error of the measurements. Significantly less swelling is recorded in all the other solvents, although the swelling degrees are still comparatively high for a polyimide. Swelling degrees of around 15-25\% are recorded in the alcohols. In all solvents except toluene, the swelling appears to be progressing over longer timescales, which indicates that no equilibrium is reached within this timescale. 

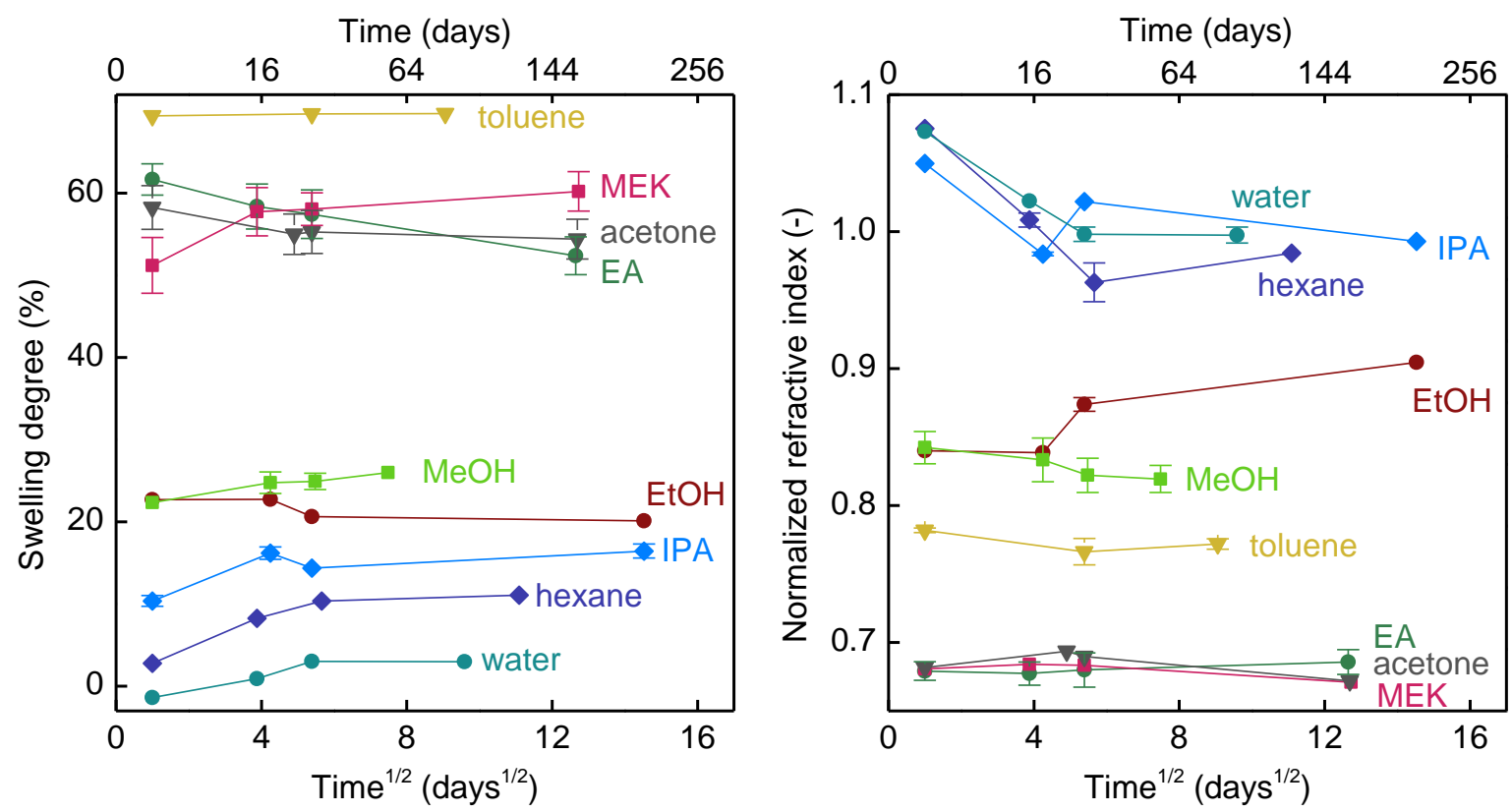

Figure 10: Swelling degree (left panel) and normalized refractive index (right panel) of Matrimid as a function of time in the solvent. Matrimid dissolved in DCM.

\section{Short-term dynamics}

Figure 11 shows the swelling (left panel) and normalized refractive index (right panel) of Matrimid layers during the first 10 hours after solvent exposure. In the aprotic polar ketones (EA, MEK, and acetone), the initial swelling is particularly fast. They are characterized by a solvent front moving through the layer, following the Case II-diffusion mechanism followed by a relaxation as a result of overshoot-dynamics (CIIo, see paragraph 2.4.2). After the nearly instant swelling, the subsequent decrease in thickness and increase in the refractive index are related to the relaxation of the polymer chains, which results in a densification of the swollen polymer matrix. Whereas the swelling degree in toluene is similar to that in the ketones, the layer follows a different path to reach this swelling degree, not showing a Case II-diffusion mechanism. The normalized refractive index of toluene higher than 1 indicates that initially, the layer is filled with solvent without displaying a corresponding swelling. Next, swelling according to a Fickian diffusion mechanism follows, after which the layer shows negative relaxations $(\mathbf{F r n f})$. The same mechanism starting with free-volume filling is also recorded in the alcohols and hexane (Frnf). 

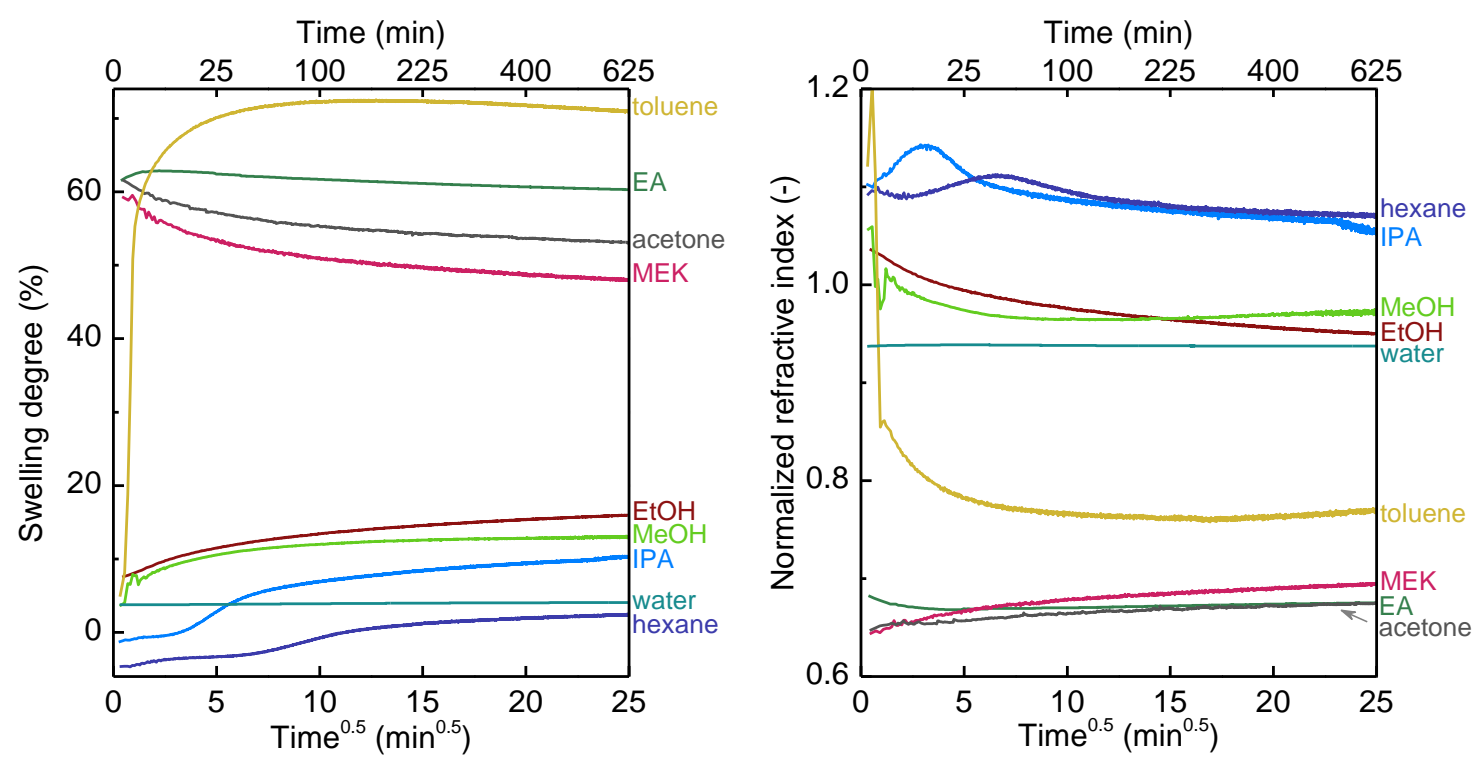

$\Delta \mathrm{SD} /(\mathrm{SD}+1)=0.12(\mathrm{MeOH}), 0.09$ (acetone), 0.06 (EA), <0.05 (other solvents). $\Delta n_{\text {norm }}=0.05$ (EA), $<0.02$ (other solvents).

Figure 11: Swelling dynamics of Matrimid as a function of time for the first hours of swelling, given by the swelling degree (left panel) and the normalized refractive index (right panel).

\section{Final remarks on Matrimid}

In conclusion, Matrimid shows a high propensity towards swelling in most of the studied solvents. Although multiple studies on Matrimid in OSN and pervaporation have been performed (see an overview in [1]), no quantitative data on the swelling of Matrimid in solvents has been found. Previous observations that Matrimid swells readily, are confirmed by the data in this study. The high swelling of Matrimid results in high solvent fractions inside the layer (see Table 4), which is further evident from the filling of the free volume in the material. Although this might be reflected by very high solvent permeances, the high solvent fraction is likely detrimental to the separation performance of the layers because of the strong swelling of the material. Cross-linking of the polymer or blending it with another polyimide such as P84 may restrict the swelling to some extent, albeit at the cost of lower solvent permeation.

\subsection{PEBAX}

PEBAX (produced by Arkema) is a block copolymer with alternating soft polyether and rigid polyamide blocks, forming a polymer with rubbery domains that can be considered physically tethered by the glassy domains. The rubbery domains give the polymer sorption properties for volatile molecules. The PEBAX polymers are available with a variety of blocks and block lengths. The ratio of the polyamide to the polyethylene block determines the 'glassiness' of the material. As a membrane, it mainly has been applied for a water vapor [86] or gas 
permeation [87] applications. It has also been suggested for deacidifying of vegetable oils, where it was used in hexane, acetone or alcoholic media [88].

\section{Long-term swelling}

Figure 12 shows the long-term development of the swelling (left panel) and normalized refractive index (right panel) of PEBAX over a measurement period of over 2 months. In all solvents, a pronounced swelling is observed. Extreme swelling is observed in DCM, in which PEBAX swells to nearly 4 times its original thickness. In the aprotic polar solvents EA, MEK and acetone, PEBAX shows swelling degrees of 50-70\%. In these solvents, the simultaneous drop in the swelling degree and the normalized refractive index can be indicative for the dissolution (D) of the polymer in the solvent. In the alcohols, PEBAX also swells strongly. In both methanol and ethanol, a possible dissolution behavior similar to that of the aprotic polar solvents is seen (D). In IPA, however, the swelling progresses over the course of 4 months. In hexane, a swelling of nearly $30 \%$ without any relaxations is recorded. PEBAX did not swell in water. In toluene, the swelling could not be measured because of the lack in optical contrast between $\operatorname{PEBAX}(n=1.49)$ and toluene $(n=1.49)$.

The dissolution of the layers is confirmed by measurements of the samples thickness after full drying, which showed that the thickness of the exposed layer lies significantly below its original thickness (reduced to $\sim 60 \%$ of the initial thickness for DCM, EA and MEK, and $\sim 80 \%$ for acetone and methanol. Here, it must be noted that a block copolymer such as PEBAX could rearrange on a supramolecular level upon drying from the solvent and may therefore result in different thicknesses after drying even without dissolution. 

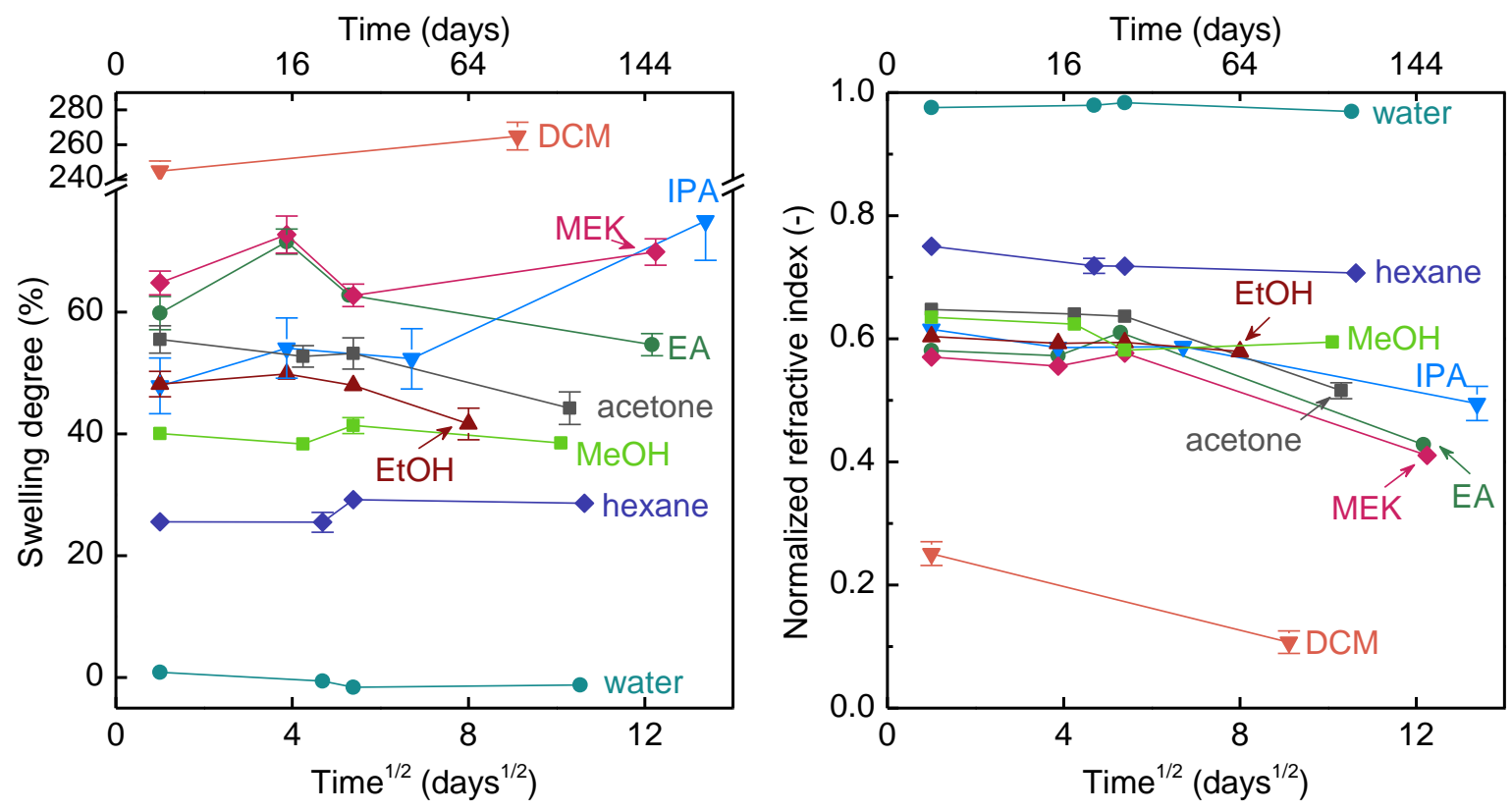

Figure 12: Swelling degree (left panel) and normalized refractive index (right panel) of PEBAX as a function of time in the solvent. The swelling of PEBAX in toluene could not be measured due to the identical refractive indices: $n_{\operatorname{PEBAX}}(1.49)=n_{\text {toluene }}(1.49)$.

\section{Short-term dynamics}

Figure 13 shows the swelling (left panel) and normalized refractive index (right panel) of the PEBAX layers during the first 10 hours after solvent exposure. In all solvents except water, PEBAX shows an instantaneous and strong swelling, resembling the behavior of rubbery swelling (R). This instantaneous swelling is followed by a gradual further increase in the swelling degree over the course of hours, indicating positive relaxations $\left(\mathbf{r}_{\mathbf{p}}\right)$, a behavior that is absent for rubbery materials. The normalized refractive indices follow trends that mirror the trend in the swelling degrees, which is as expected for a polymer with limited excess free volume. These positive relaxations presumably take place in the glassy domains. Since the rubbery domains already allowed the solvents to encompass all glassy domains, diffusion limitations are absent. Only in the case of hexane this gradual increase is not seen. 

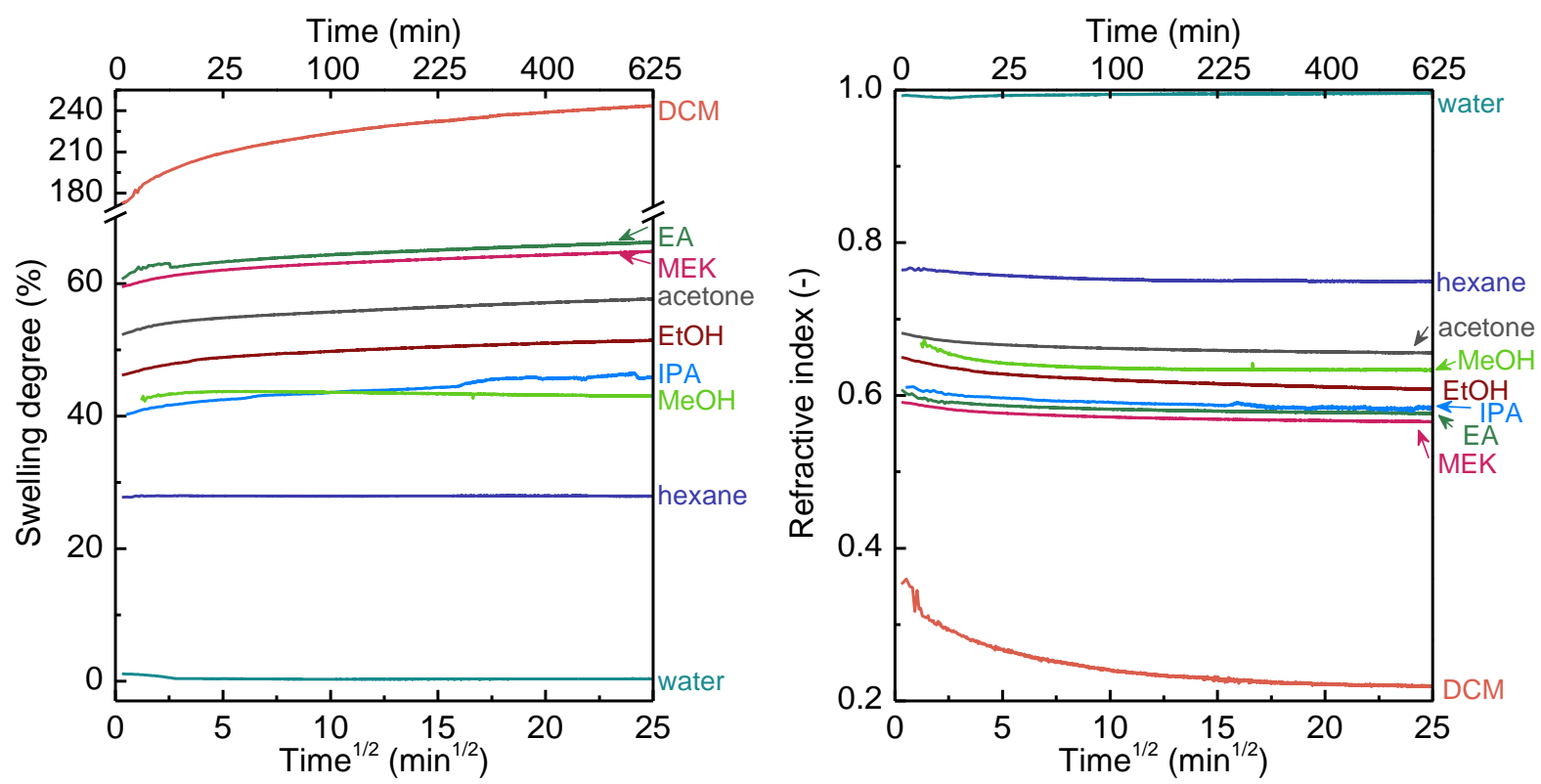

Figure 13: Swelling dynamics of PEBAX as a function of time for the first hours of swelling, given by the swelling degree (left panel) and the change in refractive index (right panel).

\section{Final remarks on PEBAX}

The combined glassy-rubbery structure of PEBAX is reflected in its swelling properties, which appear to be a superposition of rubbery and glassy behavior. On short timescales, the rubbery behavior dominates, whereas on longer timescales, the slow glassy behavior prevents the material from reaching an equilibrium swelling level. In the aprotic polar solvents and in the alcohols, slow dissolution of the layers is apparent over the course of the measurements.

\subsection{PTMSP}

Poly[1-(trimethylsilyl)-1-propyne] (PTMSP) has originally been developed as a gas separation membrane with very high permeabilities [89]. It has been a subject of many studies because of its exceptionally high free volume [90]. This free volume gives PTMSP membranes their high permeances, but also makes them prone to aging, directing research into preventing ageing in PTMSP [91]. PTMSP has been considered as an active layer for nanofiltration of ethanolic media $[92,93]$.

\section{Long-term swelling}

Figure 14 shows the long-term development of the swelling (left panel) and normalized refractive index (right panel) of PTMSP over a measurement period of over 4 months. PTMSP dissolved (D) in toluene, hexane and DCM. In the aprotic polar solvents, the swelling is strong, recording swelling degrees of 50-75\%. After the initial swelling, only acetone shows positive relaxations $\left(\mathbf{r}_{\mathbf{p}}\right)$. The most surprising behavior, however, is recorded 
in the alcohols: in water and methanol, PTSMP only swells limitedly, whereas in ethanol a much stronger swelling of 50\% is recorded, and IPA even tops the swelling of PTMSP, inducing swelling degrees well over $100 \%$. It appears that the increasingly longer hydrophobic alkane tail of the alcohols has a strong impact on the swelling. This is in line with the observation that PTMSP dissolves in apolar solvents.

PTMSP has a very high excess free volume. Even though water and methanol only limitedly swell PTMSP, the solvents intrude the polymer and cause an increase in its refractive index through the filling of the excess free volume. The higher free-volume filling of PTMSP by methanol than by water indicates that the affinity of PTMSP for methanol is higher than that for water. Acetone also displays a free-volume filling, even at a swelling level of $30-50 \%$. At such swelling levels, the dilation normally outweighs the effect of free-volume filling.

Arguably, some relaxations of PTMSP may be inferred from the data, but their magnitude is in any case very small in comparison to the other glassy polymers. This absence is uncommon, since PTMSP is considered a super-glassy material. These absent relaxations, characteristic of rubbery materials were observed for PTMSP in the past and explained by the high excess free volume inside the material [94]. We however believe that PTMSP undergoes an immediate solvent-induced glass-to-rubber transition upon solvent exposure. The transition is a result of the high solvent fraction inside the layer, which changes the material into what we came to name a 'glubber': a material demonstrating glassy characteristics in the dry state, but showing strong rubbery characteristics after solvent exposure.

The response of the PTMSP to solvent exposure cannot be grouped into solvent classes. In the solvents MEK, EA, methanol and water, the swelling achieved a stable value almost instantaneously. In IPA, ethanol and acetone, however, the swelling of the PTMSP progressed over 10 hours after an initial rapid swelling. In nearly all solvents, the swelling reached equilibrium after one day. Only in the case of acetone does the swelling progress steadily over time. The high value for the normalized refractive index in acetone $\left(n_{\text {norm }}>1\right)$ is uncommon for such high swelling levels and could indicate an extreme free-volume filling by acetone. 

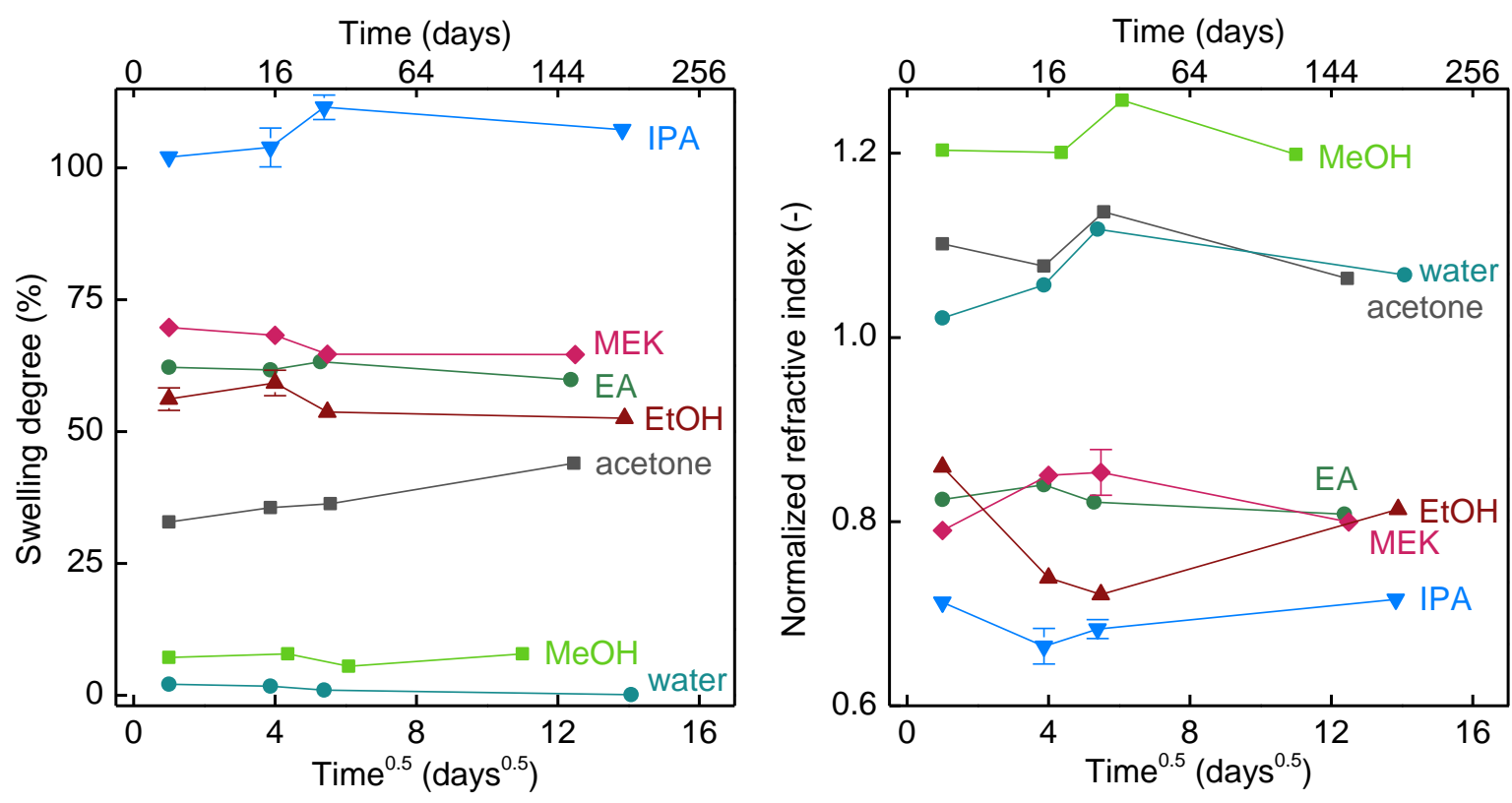

Figure 14: Swelling degree (left panel) and normalized refractive index (right panel) of PTMSP as a function of time in the solvent. PTMSP dissolved in toluene, hexane and DCM.

\section{Short-term dynamics}

Figure 15 shows the swelling (left panel) and normalized refractive index (right panel) of PTMSP layers during the first 10 hours after solvent exposure. Instantaneously after exposure to the solvent, PTMSP shows a strong swelling with nearly rubbery characteristics, directly followed by a continuing swelling through positive relaxations $\left(\mathbf{F r}_{\mathbf{p}}\right)$. The changes in the normalized refractive index indicate that filling of the free volume in PTMSP by methanol, ethanol, and acetone is not compensated by layer swelling (see also Section 2.2.2). For the other solvents, the drop in the normalized index is lower than would have been expected on the basis of the swelling of the material, indicating a high likeliness for filling of the free volume of the material for these solvents as well $\left(\boldsymbol{F r}_{\mathbf{p}} \mathbf{f}\right)$. 

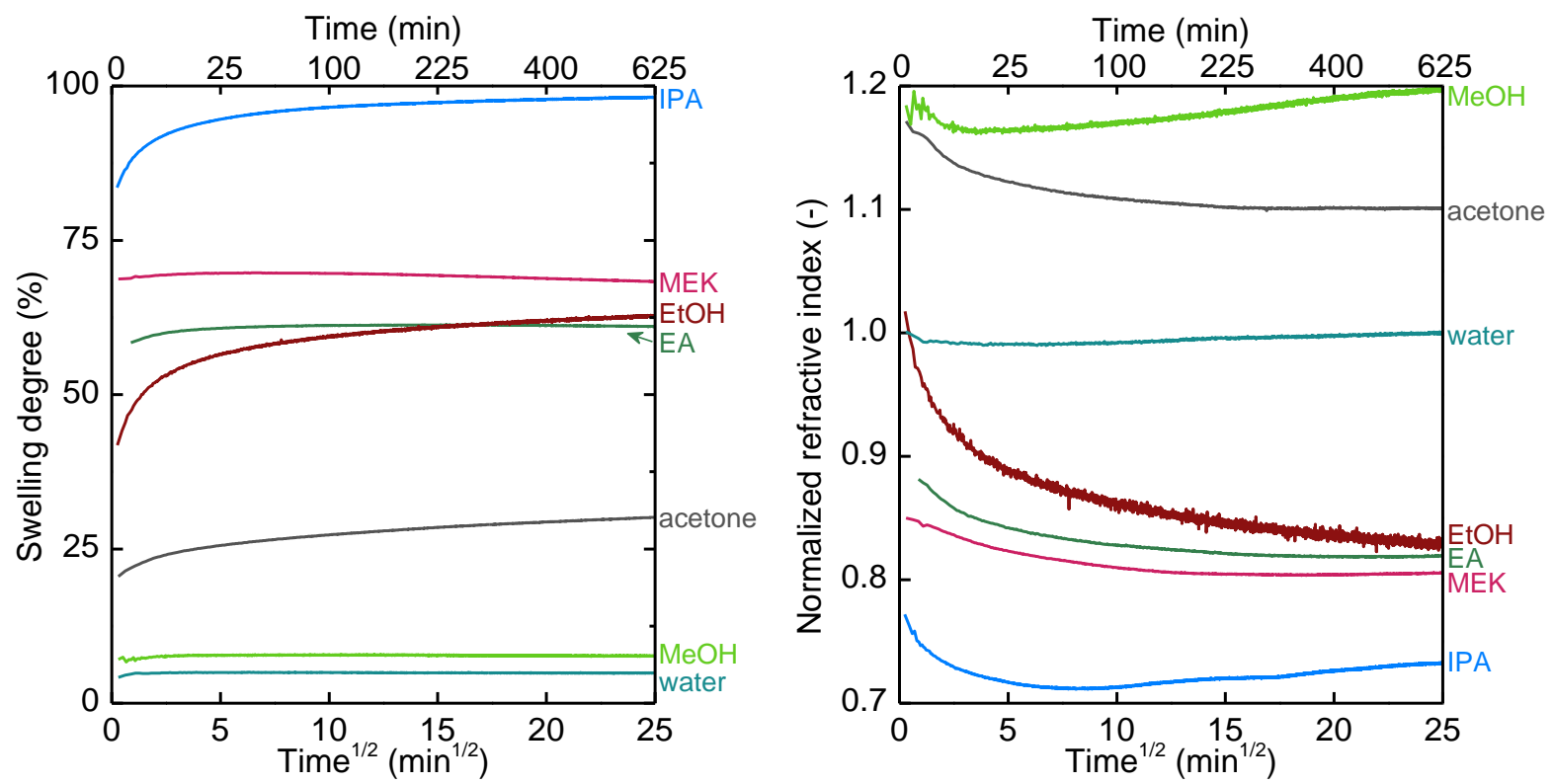

Figure 15: Swelling degree of PTMSP as a function of time for the first hours of swelling, given by the swelling degree (left panel) and the change in refractive index (right panel).

\section{Final remarks on PTMSP}

It can be concluded that the rapid initial swelling of PTMSP, combined with the short timescale in which the equilibrium swelling is attained, shows close resemblance to the behavior of a rubbery polymer. Although strong ageing effects are recorded for the dry layers of PTMSP, as was evident from differences in the refractive index of the dry layers. These effects disappear at the moment the PTMSP is brought into contact with a solvent. We suggest that for application of PTMSP in organic solvents, the material should be considered a 'glubber': a glassy material in the dry state that behaves as a rubbery polymer when in contact with solvents.

\subsection{Polysulfones}

Polysulfones are glassy polymers that are used as a support for OSN membranes. In the 1960s, PSf has been introduced as a hydrophobic substitute for cellulose acetate membranes in hemodialysis [95] and has been suggested for applications in gas separation in [64]. The widespread application of PSf membranes has mainly been due to their easy processability and good resistance to a wide range of pHs [96]. PES membranes find their use in a broad range of (tight) ultrafiltration processes including drinking water treatment, dairy applications, beer filtration and many others [97]. However, PES membranes have been considered for organic solvent applications as well $[98,99]$. 
In NF and RO applications, both PES and PSf currently find their use as a support layer for interfacial-polymerization membranes $[1,100]$. Both polymers are susceptible to solventinduced changes at high pressures. For alcohols, some applications using classic interfacially polymerized nanofiltration membranes are reported, which suggests that PES/PSf is suitable as support in these relatively mild environments [101,102].

\subsubsection{PES}

\section{Long-term swelling}
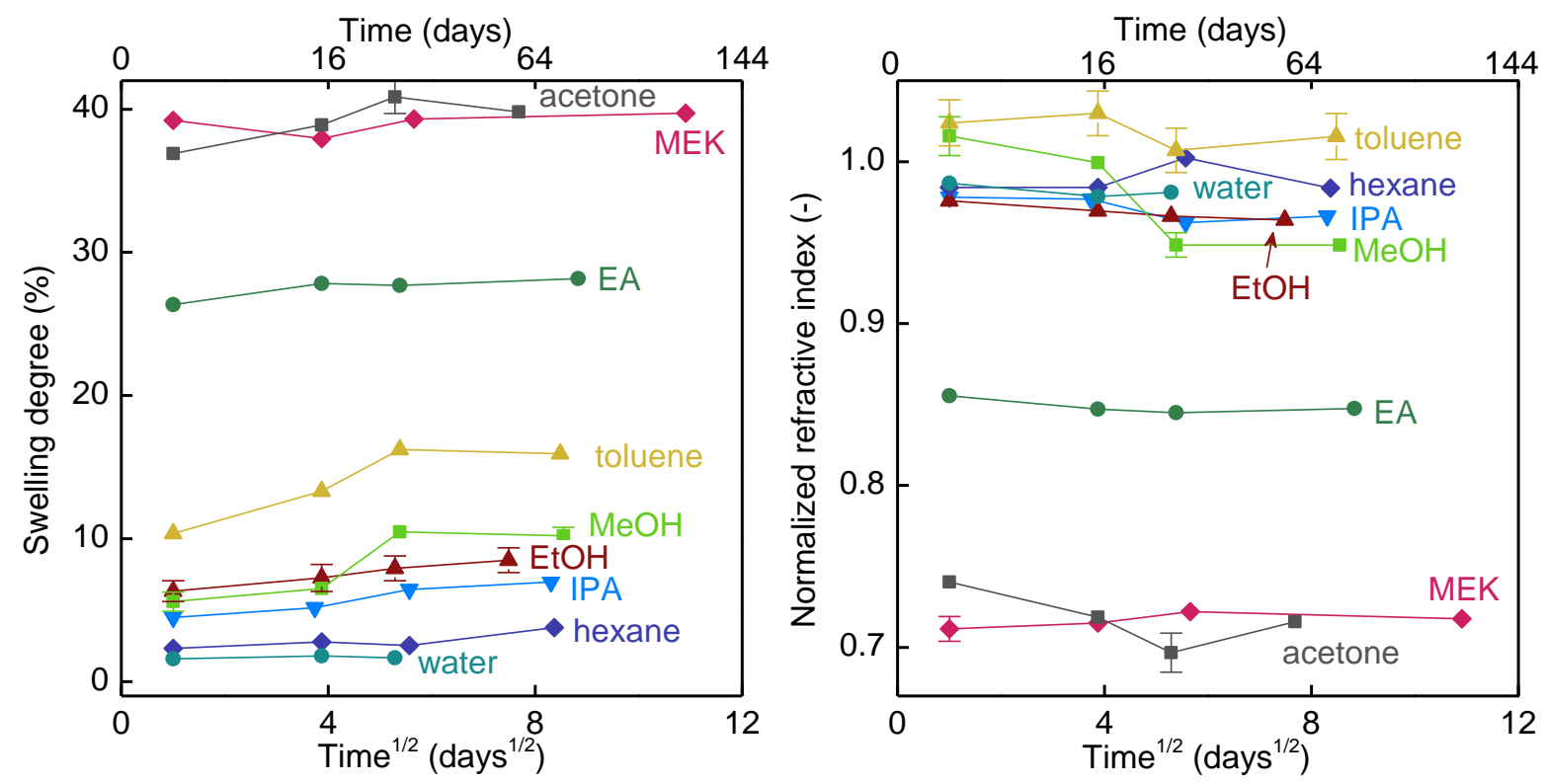

Figure 16 shows the long-term development of the swelling (left panel) and normalized refractive index (right panel) of PES over a measurement period of over 2 months. In DCM, PES dissolved (D). The swelling is most pronounced in the aprotic polar ketones. In the third aprotic polar solvent, EA, the swelling is already less pronounced. In all the other solvents, the swelling degrees are much lower and increasing slightly in time. The normalized refractive indices are consistent with the swelling data. In the case of toluene, $n_{\text {norm }}$ exceeding 1 suggests a free-volume filling; however, the large error bars indicate a strong uncertainty on the data values. 

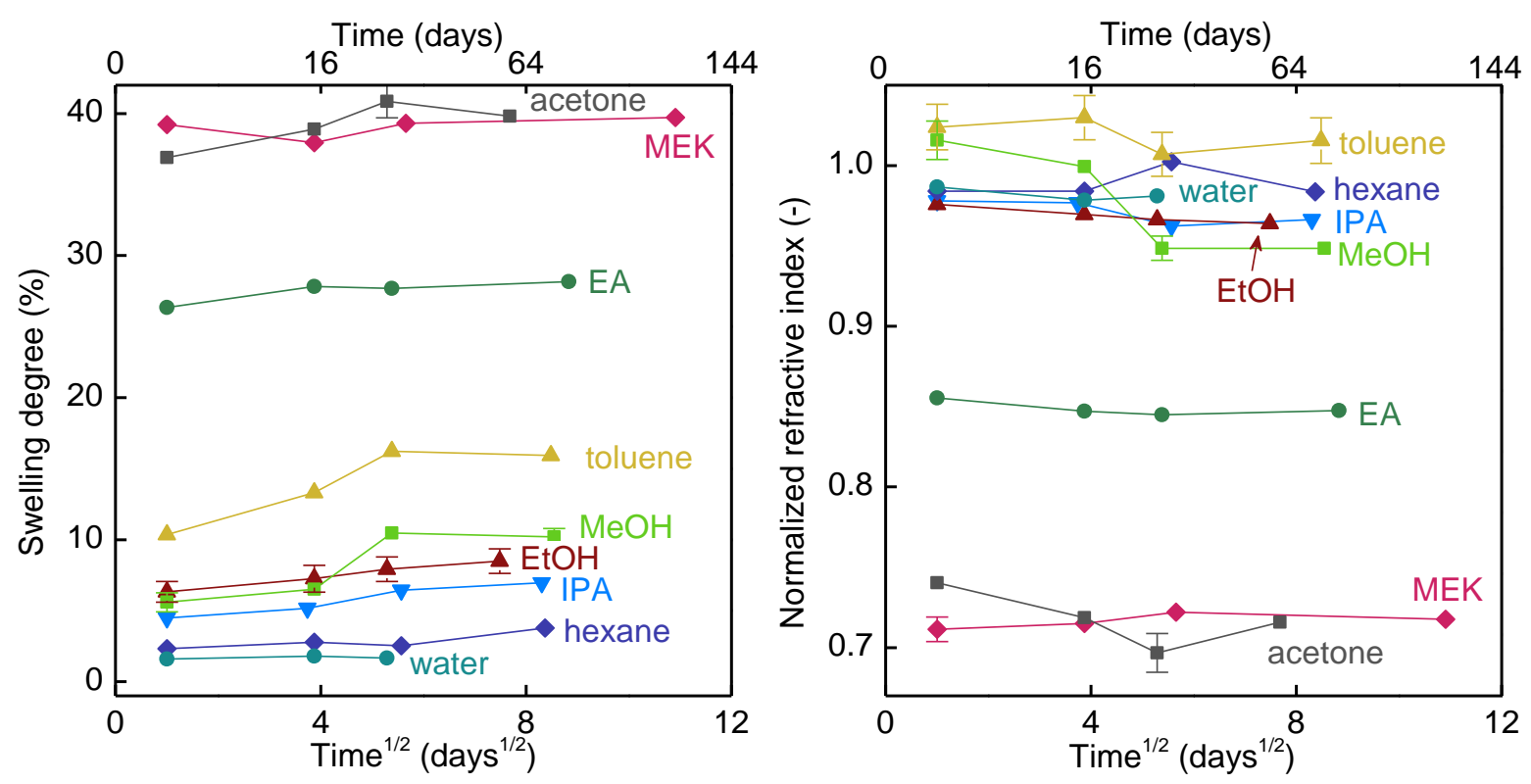

Figure 16: Swelling degree (left panel) and normalized refractive index (right panel) of PES as a function of time in the solvent. PES dissolved in DCM.

\section{Short-term dynamics}

Figure 17 shows the swelling (left panel) and normalized refractive index (right panel) of PES layers during the first 10 hours after solvent exposure. For the ketones MEK and acetone, the swelling occurs through a Case II-diffusion mechanism combined with overshoot dynamics (CIIo). As a consequence, the short-term swelling degree after a few minutes is higher than the values recorded on longer time scales. The third aprotic polar solvent, EA, the swelling follows a Fickian diffusion with slight positive relaxation $\left(\mathbf{F r}_{\mathbf{p}}\right)$. In all the other solvents, the swelling also follows the $\mathbf{F r}_{\mathbf{p}}$-mechanism.

It is noted that for EA, toluene, IPA and hexane, the data could not be modelled accurately using a simple isotropic film model; for these datasets, a gradient in the refractive index was found to best model the data. As for the long-term data, the normalized refractive indices are consistent with the swelling data. As described for the long-term data, the error bars for toluene are significant and the absolute values of the data should be interpreted with care. However, the trend in the normalized refractive index for the short-term data supports the theory that free-volume filling of PES by toluene takes place. 

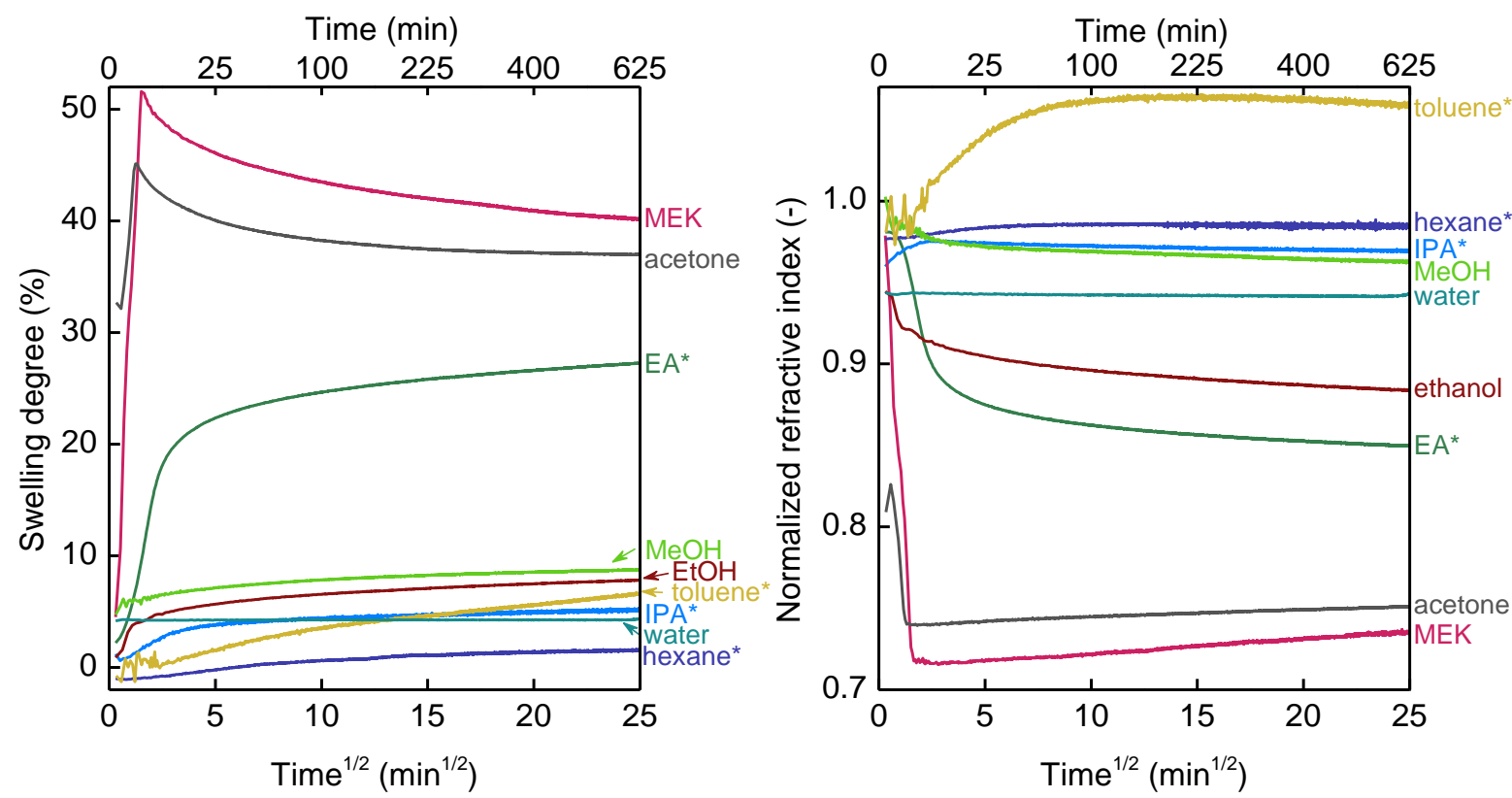

Figure 17: Swelling dynamics of PES as a function of time for the first hours of swelling, given by the swelling degree (left panel) and the change in refractive index (right panel).

\section{Final remarks on PES}

In conclusion, the resistance of PES to swelling is poor in aprotic polar solvents (ketones) and decent in all others. In long-term operation, the continuous swelling of the polymer matrix in the presence of alcohols and apolar solvents potentially impacts membrane properties. 


\subsubsection{PSf}

\section{Long-term swelling}

Figure 18 shows the long-term development of the swelling (left panel) and normalized refractive index (right panel) of PSf over a measurement period of 2 months. In DCM, PSf dissolved (D). The swelling behavior of PSf with respect to solvent type is to a large extent similar to that of PES: the ketones induce a significant swelling of the PSf, with swelling values of $30-40 \%$ and the alcohols, water, and hexane induce limited swelling $(<10 \%)$. For PSf, the trends in the normalized refractive index are all in line with the swelling degree.

However, in sharp contrast to PES, toluene swells PSf significantly, inducing swelling degrees of over $60 \%$. PES was swollen by toluene to only $15 \%$ after two months. Noteworthy as well is the fact that the swelling induced by acetone and EA is slightly lower than that induced by MEK, whereas for PES, the swelling induced by MEK and acetone was similar, and EA displayed slightly lower swelling degrees.
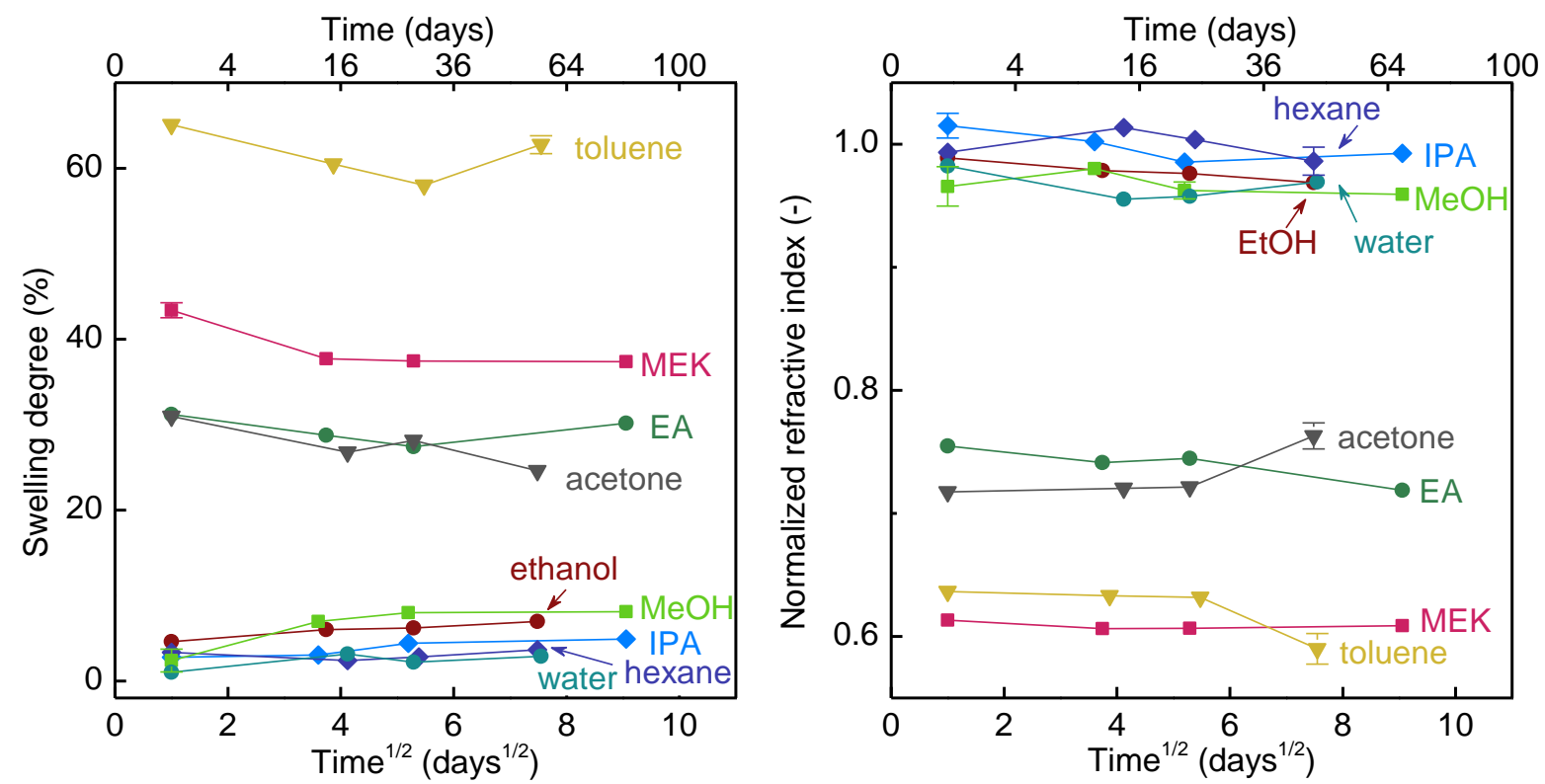

Figure 18: Swelling degree (left panel) and normalized refractive index (right panel) of PSf as a function of time in the solvent. PSf dissolved in DCM. 


\section{Short-term dynamics}

Figure 19 shows the swelling (left panel) and normalized refractive index (right panel) of P84 layers during the first 10 hours after solvent exposure. Again, except for toluene, the swelling behavior of PSf is similar to that of PES, displaying strong swelling following the Case IIdiffusion with overshoot dynamics (CIIo) in the ketones and relatively minor swelling in the alcohols, water, and hexane, where a Fickian diffusion with positive relaxations is recorded $\left(\mathbf{F r}_{\mathbf{p}}\right)$. For toluene, the swelling dynamics appear to be similar to that of the ketones, also following a Case II-diffusion mechanism (CIIo). The unexpected behavior of the normalized refractive index can be attributed to the high refractive index of toluene in combination with the used measurement cell, which is known to result in a high susceptibility of the experiment to external perturbations, such as small temperature variations [62]. It could be argued that for the polar aprotic solvents, the absence of an increase in the normalized refractive index during the apparent overshoot dynamics would hint at a slow dissolution of these polymers. However, the long-term data given in Figure 18 does not support this conclusion. In addition, measurements of the sample thickness after drying showed no indications of dissolution.
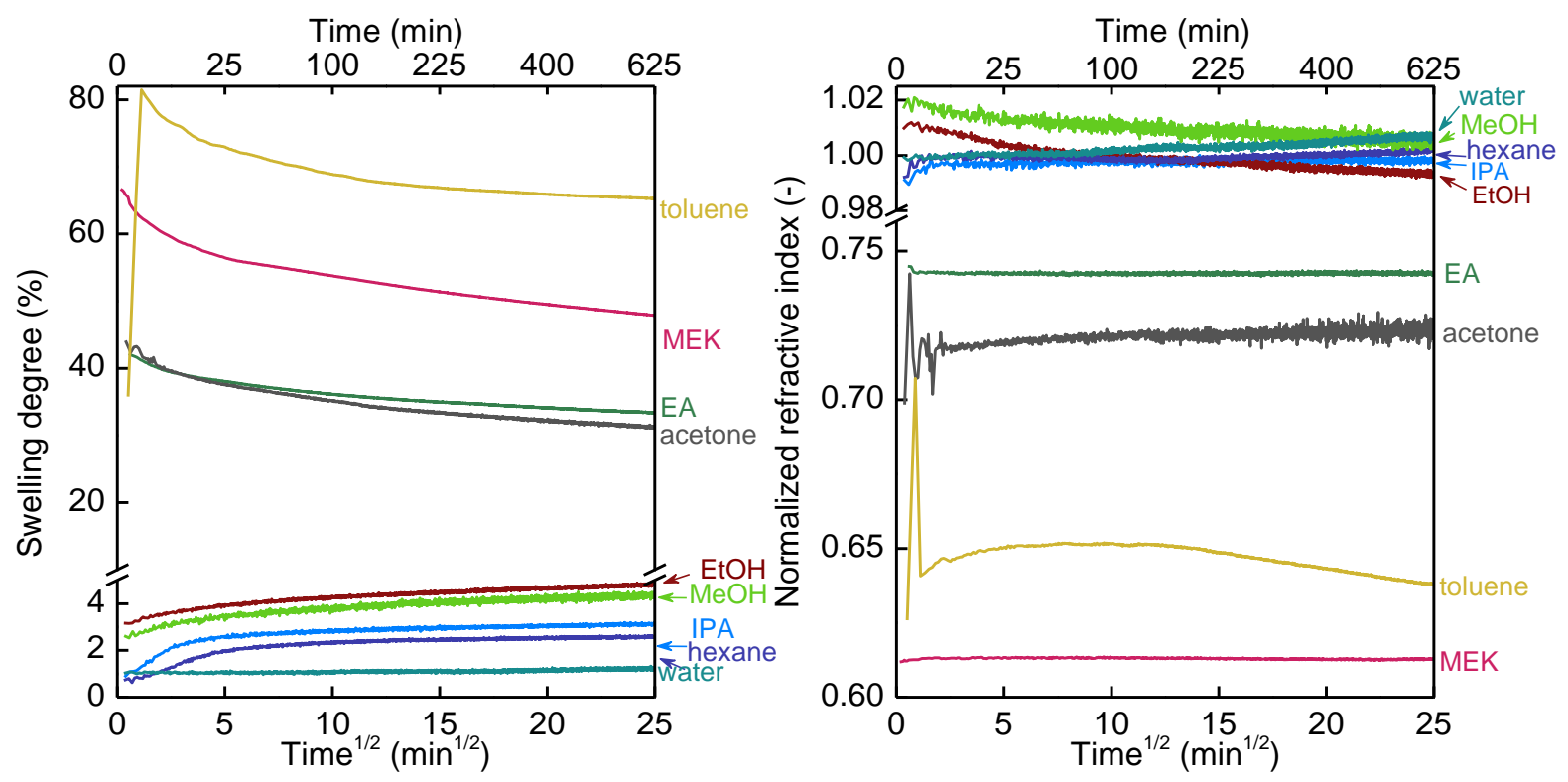

Figure 19: Swelling dynamics of PSf as a function of time for the first hours of swelling, given by the swelling degree (left panel) and the normalized refractive index (right panel).

\section{Final remarks on PSf}

In conclusion, PSf has a poor stability in ketones and toluene, following a Case II-diffusion swelling (CIIo). Especially the remarkably different behavior in toluene compared to PES is an important learning. In nanofiltration and reverse osmosis membranes, typically PSf is 
applied as a support structure [103], due to the better properties achievable with this polymer. Contrary to others [104], we do not see significant differences in the swelling of PSf and PES in alcohols. The presence of toluene can have dire consequences for membrane performance. It is known in industry that undesired membrane behavior is registered in the presence of even low levels(ppms) of aromatics. In the presence of alcohols, the layers do not reach a stable swelling over the course of months, indicating that the long-term operation of PSf-membranes could be unstable in the presence of these solvents.

\subsection{PAN}

Poly(acrylonitrile) (PAN) is a polymer that is widely used as an ultrafiltration membrane in aqueous applications, because it is more hydrophilic than most organic polymers and has sufficient chemical resistance to remain stable in aggressive cleaning environments [105]. In NF applications, PAN is mainly used as a support [106], because of the difficulties in processing it into a high-quality nanofiltration membrane. Depending on the application, PAN can be modified to make it more hydrophilic or hydrophobic [107-109]

\section{Long-term swelling}

Figure 20 shows the long-term development of the swelling (left panel) and normalized refractive index (right panel) of PAN over a measurement period of more than 2 months. The main observation for PAN is the very limited swelling in all solvents, which is unsurprising considering the low solubility of the polymer in many common solvents [106]. With a maximum swelling of nearly $10 \%$ in methanol after 2.5 months, it swells more than a factor 2 less than PEI does. In toluene and DCM, some swelling to 6 and $4 \%$ respectively is displayed as well. In all other solvents, the swelling is $\leq 1 \%$. Except for methanol and toluene, the changes in the normalized refractive index are near zero, especially considering the uncertainty in the measurements due to the small changes. In methanol, the normalized refractive index decreases due to the swelling of the layer. In toluene, an increase in the normalized refractive index is registered, which is unexpected considering the low excess free volume of PAN. However, since the optical contrast between PAN $(n=1.51)$ and toluene $(n=$ 1.49) is low, the experimental uncertainty is significant. In water, the PAN layer delaminated. 

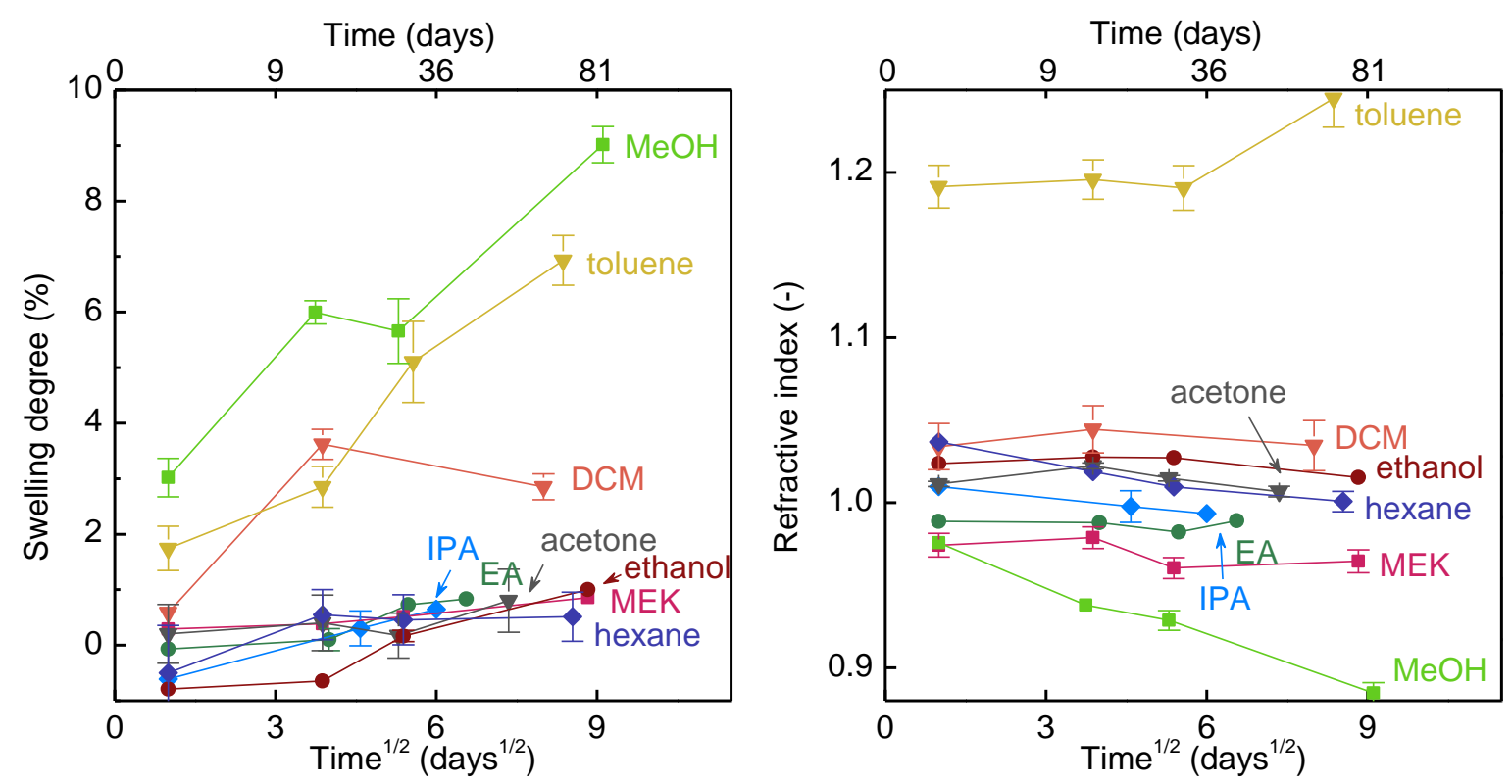

Figure 20: Swelling degree (left panel) and refractive index (right panel) of PAN as a function of time in the solvent. The PAN layer delaminated in water.

\section{Short-term dynamics}

Figure 21 shows the swelling (left panel) and normalized refractive index (right panel) of PAN layers during the first 10 hours after solvent exposure. The strong noise on the data here is due to the low signal-to-noise ratio, caused by the marginal swelling of PAN. Because of this limited swelling, the data are highly susceptible to slight perturbations of the system such as minor temperature variations and as such, the authors recommend against overinterpretation of the trends. In water, the PAN layer delaminated within hours after immersion. 

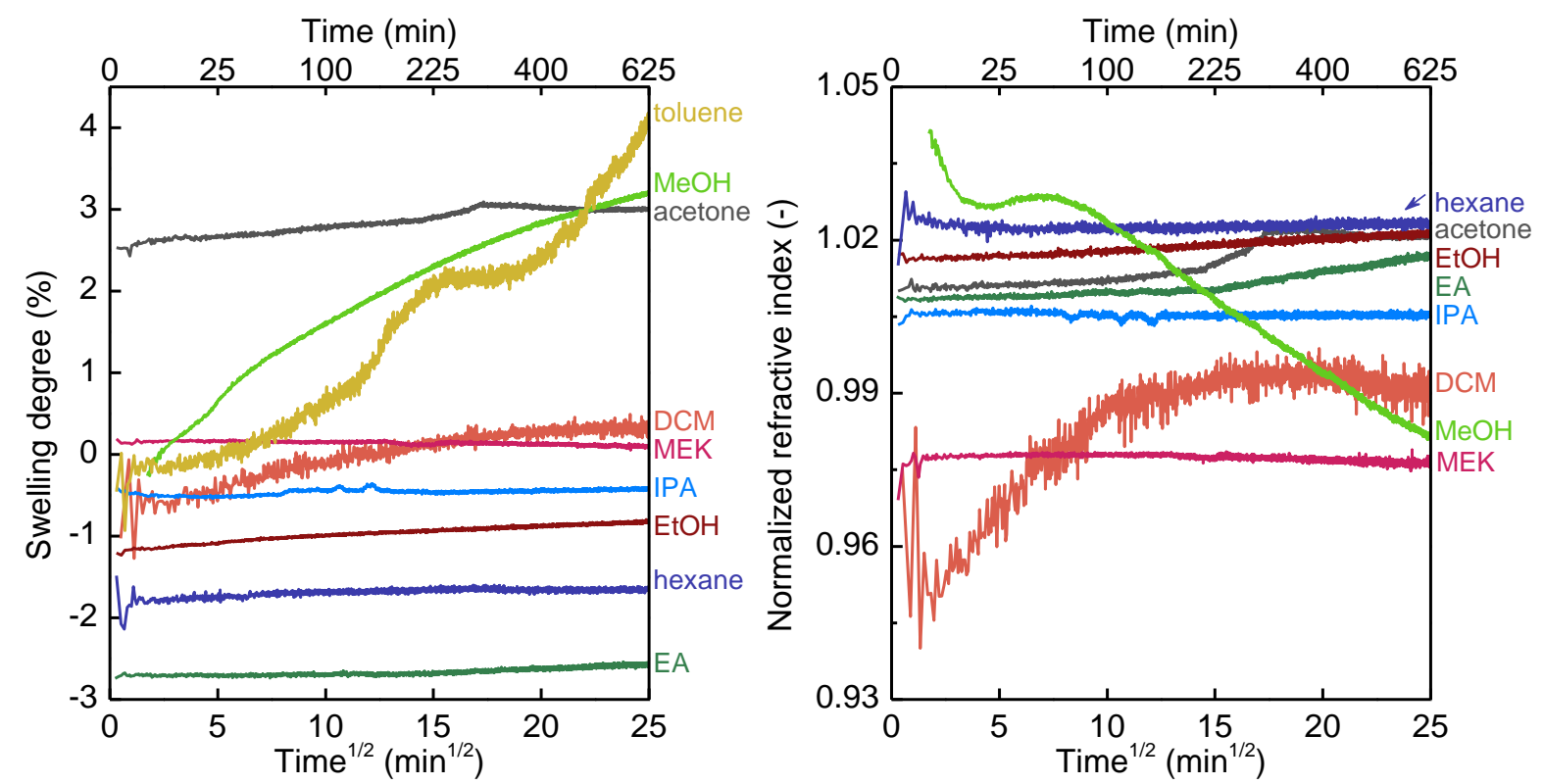

Figure 21: Swelling dynamics of PAN as a function of time for the first hours of swelling, given by the swelling degree (left panel) and the change in refractive index (right panel). Note that the apparent strong scatter on the data is due to limited $y$-axis range. Because of the low optical contrast, the refractive index data for PAN-toluene shows very strong scattering and is not shown here (the data are given in the Supplementary Information).

\section{Final remarks on PAN}

It can be concluded that PAN is resistant to a wide range of solvents, as its swelling remains marginal even over long timescales. The low fractional free volume in PAN prevents solvent uptake and plasticization of the polymer matrix. Table 4 in the Conclusions shows the solvent volume fractions inside the swollen layer. Due to the limited swelling, the solvent volume fractions are very low and in most cases negligible. As a result, dense PAN layers show very low permeances and are unsuited as nanofiltration membranes. These properties are, however, beneficial in porous support layers, where the solvent permeate through the pores instead of through a dense solvent-filled layer, explaining the wide use of PAN for these applications. 


\section{Discussion \& Reflections}

The interactions of 10 organic solvents (hexane, toluene, dichloromethane, ethyl acetate, methyl ethyl ketone, acetone, isopropanol, ethanol, methanol, water) with 9 polymers commonly used for organic solvent nanofiltration (P84, Matrimid, PEI, PAN, PES, PSf, PEBAX, PTMSP, PDMS) were studied. The obtained results demonstrate that the swelling behavior of polymers is complex for three reasons:

1. For many polymers, the swelling changes over long timescales of over 2 months. The results obtained on short timescales do not always allow for direct extrapolation to longer time scales.

2. Structural similarity of polymers does not guarantee similar swelling behavior;

3. Swelling behavior of solvents cannot solely be explained by classifying solvents into aprotic, polar aprotic and polar protic solvents.

General behaviorAnother possible visualization of the affinity of the polymers for the studies solvents is a plot of the swelling degree versus the Hildebrand solubility parameter $(\delta)$, a parameter that is typically used to estimate solubility of polymers in solvents. This representation and a discussion of these data are given in the Supplementary Information.

shows an overview of the swelling degrees obtained after long-term solvent exposure. The diagram allows for quick extraction of the main trends. 


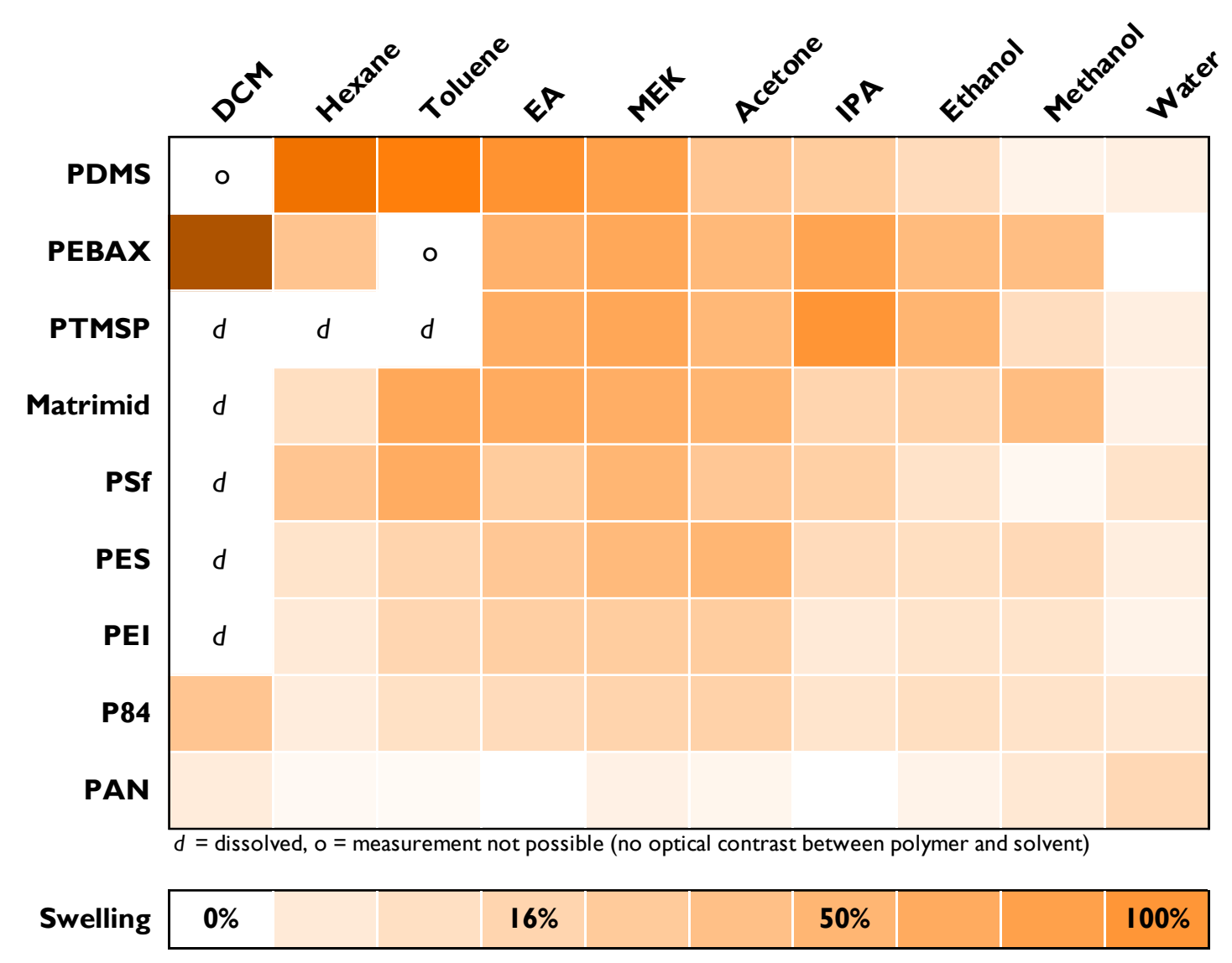

Figure 22: Overview of all swelling degrees measured after long-term exposure to the respective solvents. For each polymer-solvent combination, the last measured data point was taken. ' 0 ' indicates absence of a measurement due to lack of optical contrast, ' $d$ ' indicates dissolution of the polymer in the solvent.

The polymers can be mainly classified into four groups: rubbery behavior (PDMS and PEBAX), glubbery behavior (PTMSP), glassy behavior (Matrimid, PSf, PES, PEI, and P84) and non-swelling (PAN). PDMS is a well-understood rubbery polymer. As such, the data presented here are not surprising. The measurements performed here using spectroscopic ellipsometry agree well with values obtained previously using other measurement methods $[12,66]$ and demonstrate the high quality data that can be obtained. Understanding the behavior of PAN is also straightforward: it shows limited swelling in a wide variety of solvent classes.

The classification of PEBAX and PTMSP can be debated: on one hand, due to the glassy domains, the behavior of PEBAX is not fully rubbery, since its swelling exhibits some temporal changes initially; and on the other hand, PTMSP, a well-known glassy polymer 
shows some typical rubbery characteristics after being exposed to a strong solvent: a behavior we came to call 'glubbery' (glassy + rubbery).

The other polymers show behaviors typical of glassy polymers. The very glassy behavior of the polyimides, especially P84 and PEI, is confirmed by their limited swelling and strongly time-dependent behavior, even on very long timescales of over 1 month. Although Matrimid is also a polyimide, its susceptibility to swelling is much higher, resulting in high swelling degrees of $60-70 \%$ in toluene and the aprotic polar solvents.

PES and PSf are two polymers that are highly interesting from an academic perspective. The difference in molecular structure is limited (PSf contains a dimethyl group instead of a sulfone group every other repeating unit, see Table 1), but their properties are, in some cases, vastly different. Especially in toluene, the swelling of PES is small, but the swelling of PSf is strong. For all other solvents, the swelling behavior can be considered comparable. It appears that the dimethyl group in PSf allows for the penetration of toluene into the polymer's structure.

Looking at the data grouped by solvent, some interesting trends can be identified. The chlorinated DCM dissolves nearly all polymers and only PAN does not swell significantly in DCM. For the other two apolar solvents, hexane and toluene, it is observed that the aromatic toluene in all cases induces a stronger swelling than the aliphatic hexane, which presumable originates from the affinity of toluene to the abundant aromatic and unsaturated bonds in the polymers. The polar aprotic solvents EA, MEK, and acetone typically induce significant swelling as well. Generally, but not always, MEK displays the strongest swelling, followed by EA and acetone. Only in the case of PES, acetone induces a stronger swelling than EA. Not always do these three solvents display the same swelling mechanism. Taking PES as an example, the swelling in MEK and acetone follows the dynamics of a Case II-diffusion with overshoot dynamics (CIIo), whereas the swelling in EA follows a Fickian diffusion with positive relaxation $\left(\mathbf{F r}_{\mathbf{p}}\right)$.

The alcohols typically only result in limited swelling of all polymers, PTMSP and PEBAX being notable exceptions. Typically, IPA results in the highest swelling degrees, followed by ethanol and methanol. Only for Matrimid, this trend is clearly reversed, with methanol inducing the highest swelling degrees. Water typically does not swell these polymers significantly. This behavior is expected, since the polymers were selected on their relevance 
for organic solvent nanofiltration, for which an affinity to organic solvents rather than water is desired.

All swelling data are summarized in the graphical abstract. Another possible visualization of the affinity of the polymers for the studies solvents is a plot of the swelling degree versus the Hildebrand solubility parameter $(\delta)$, a parameter that is typically used to estimate solubility of polymers in solvents. This representation and a discussion of these data are given in the Supplementary Information.

\subsection{Solvent volume fractions}

Quantitative data aids in the development of predictive models of the performance of polymers under application conditions. One of the most interesting quantitative parameters that can be derived is the solvent volume fraction inside the swollen layers, since it relates directly to the solvent activity (see Section 2.3.1). As previously indicated, because of the presence of an excess free volume in glassy polymers, the solvent volume fractions as determined from the dilation and from the refractive index, may differ.

If a polymer contains excess free volume in its dry state, it is impossible from the (dilatational) swelling degree alone to distinguish whether the solvent fills the excess free volume without dilation (Langmuir-sorption), introduces dilation (Henry-sorption), or follows a combined mechanism. Therefore, we present the solvent fractions in the layers, calculated both from the dilation using Equation 3 and the refractive index using Equation 5. To account for the relaxation of the excess free volume in Equation 5, the refractive index of polymer was replaced with the hypothetical liquid refractive index.

To illustrate, P84 has a high free volume, which can potentially take up plenty of solvent without showing complementary swelling. In the Langmuir case, the free-volume filling should result in an increase in the normalized refractive index. In the Henry case, the normalized refractive index should decrease. In the mixed case, it can go either up or down, depending on the relative magnitude of the two contributions. In the ideal case of data without experimental uncertainty, the combination of swelling degree and refractive index can be used to calculate the solvent volume fraction in the layer.

An overview of these solvent volume fractions inside the swollen layers is given in Table 4 . Additionally, the data is available graphically in the Supplementary Information. Since the dilatational values (Henry-Sorption) do not incorporate free volume filling, they represent an 
estimate for the minimal solvent volume fractions inside the polymers. For rubbers, due to the absence of free volume, they represent the true value. For glasses, because of the free-volume filling (Langmuir sorption), the true solvent volume fractions will always be higher. In the case of high solvent-volume fractions (i.e. strongly swelling polymers), the error introduced by this calculation is minimal, since the contribution of Langmuir sorption is small in comparison to that of the Henry sorption.

Table 4: Low boundary values for the volume fractions of solvent inside the swollen layers in percent at the last measurement point, calculated from the swelling degree assuming zero excess free volume

\begin{tabular}{|c|c|c|c|c|c|c|c|c|c|c|}
\hline$\Phi_{\mathrm{S}, \min }(\%)$ & DCM & EA & IPA & MEK & Acetone & Ethanol & Hexane & Methanol & Toluene & Water \\
\hline PDMS & & $51.1 \pm 0.1$ & $26.3 \pm 0.3$ & $43.6 \pm 0.1$ & $23.4 \pm 0.2$ & $10.1 \pm 0.2$ & $64.6 \pm 0.1$ & $1.1 \pm 0.3$ & $59.5 \pm 0.2$ & $2.6 \pm 0.6$ \\
\hline P84 & $0+03$ & 11. & \pm 0.0 & \pm 0.0 & & $=0.4$ & \pm 0.1 & & 0.1 & $5.03 \pm 0.1$ \\
\hline PEI & & $16.3 \pm 0.2$ & $3.26 \pm 0.1$ & $18.2 \pm 0.1$ & $18.7 \pm 0.2$ & $3.66 \pm 0.2$ & $2.43 \pm 0.2$ & $6.53 \pm 0.2$ & $11.4 \pm 0.5$ & $0.59 \pm 0.5$ \\
\hline Matrimid & & $34.3 \pm 0.9$ & $14.1 \pm 0.6$ & $37.5 \pm 0.9$ & $35.2 \pm 1.0$ & $16.7 \pm 0.4$ & $9.96 \pm 0.0$ & 20.6 & $41.0 \pm 0.0$ & $2.91 \pm 0.4$ \\
\hline PEBAX & $72.6 \pm 0.5$ & $35.3 \pm 0.7$ & $42.8 \pm 2.1$ & $41.1 \pm 0.7$ & $30.6 \pm 1.2$ & $29.4 \pm 1.2$ & $22.2 \pm 0.4$ & $27.8 \pm 0.3$ & & $-1.2 \pm 0.8$ \\
\hline PTMSP & & $37.4 \pm 0.3$ & $51.7 \pm 0.3$ & $39.2 \pm 0.3$ & $30.5 \pm 0.5$ & $34.4 \pm 0.6$ & & $7.28 \pm 0.4$ & & $0.11 \pm 0.9$ \\
\hline PES & & $21.9 \pm 0.1$ & $6.50 \pm 0.1$ & $28.4 \pm 0.1$ & $28.4 \pm 0.1$ & $7.82 \pm 0.7$ & $3.65 \pm 0.1$ & $9.25 \pm 0.4$ & $13.7 \pm 0.3$ & $\mathrm{n} / \mathrm{a}$ \\
\hline PSf & & $23.1 \pm 0.2$ & $4.66 \pm 0.1$ & $27.1 \pm 0.1$ & & 6.4 & $3.51 \pm 0.5$ & & $38.5 \pm 0.3$ & $2.80 \pm 0.2$ \\
\hline PAN & $2.77 \pm 0.2$ & $0.82 \pm 0.1$ & & $0.85 \pm 0.1$ & $0.79 \pm 0.5$ & $0.99 \pm 0.1$ & $0.51 \pm 0.4$ & $8.27 \pm 0.2$ & $6.48 \pm 0.3$ & $11.4 \pm 2.0$ \\
\hline
\end{tabular}

\section{Conclusions}

In this paper, 90 polymer-solvent combinations have been investigated. The given data form a basis for fundamental investigations and practical application of these materials. The methodology can be applied to study further polymer-solvent combinations that are of interest. Here, the influence of parameters such as molecular weight, degree of cross-linking, blending, and solvent mixtures can be considered. To gain even deeper insight into the process occurring inside the layers, spectroscopic ellipsometry can be combined with other techniques, such as a quartz-crystal microbalance or dielectric relaxation spectroscopy. By applying the layers to porous substrates and analyzing these in situ under pressure [32], the influence of pressure gradients on the layers can be studied further.

The error bars in the data in this study are predominantly caused by the thickness inhomogeneity in the spin-coated layers. Therefore, a further improvement in data accuracy can be obtained by better controlling the layer homogeneity. The uncertainties introduced by inaccuracies in the refractive index are inherent to the applied measurement method; they can be minimized [62], but not avoided completely. Furthermore, for the glassy materials, it 
would be interesting to store the samples for much longer timescales of multiple years in order to assess the final (equilibrium) solvent concentration that is reached inside these layers.

\section{Acknowledgements}

The authors acknowledge Jeff Wood for proofreading the manuscript, Vic van Dijk for sharing his knowledge on the optimization of the spin-coating recipe for polymers dissolved in NMP, and Bart Zaalberg for aiding in the optimization of the spin-coating and ellipsometry measurement.

\section{Data Availability}

In addition to the data given in the Supplementary Information, the raw as well as the processed data required to reproduce these findings are available to download from DOI: 10.4121/uuid:c454adaa-30ea-4831-99c0-f8d5f9a624e1b.

\section{References}

[1] P. Vandezande, L.E.M. Gevers, I.F.J. Vankelecom, Solvent resistant nanofiltration: separating on a molecular level., Chem. Soc. Rev. 37 (2008) 365-405. doi:10.1039/b610848m.

[2] P. Marchetti, M.F.J. Solomon, G. Szekely, A.G. Livingston, Molecular Separation with Organic Solvent Nanofiltration - A Critical Review, Chem. Rev. 114 (2014) 10735-10806. doi:10.1021/cr500006j.

[3] J.P. Robinson, E.S. Tarleton, K. Ebert, C.R. Millington, A. Nijmeijer, Influence of Cross-Linking and Process Parameters on the Separation Performance of Poly(dimethylsiloxane) Nanofiltration Membranes, Indust. 44 (2005) 3238-3248.

[4] M.I. Artsis, A.E. Chalykh, N.A. Khalturinskii, Y.V. Moiseev, G.E. Zaikov, Diffusion of organic diluents into ethyl cellulose, Eur. Polym. J. 8 (1972) 613-626. doi:10.1016/0014-3057(72)90137-1.

[5] M. Wessling, S. Schoeman, T. van den Boomgaard, C.A. Smolders, Plasticization of gas separation membranes, Gas Sep. Purif. 5 (1991) 222-228. doi:10.1016/0950-4214(91)80028-4.

[6] W. Ogieglo, M. Wessling, N.E. Benes, Polymer Relaxations in Thin Films in the Vicinity of a Penetrantor Temperature-Induced Glass Transition, Macromolecules. (2014) 140516121450004. doi:10.1021/ma5002707.

[7] C.A. Scholes, G.Q. Chen, G.W. Stevens, S.E. Kentish, Plasticization of ultra-thin polysulfone membranes by carbon dioxide, J. Memb. Sci. 346 (2010) 208-214. doi:10.1016/j.memsci.2009.09.036.

[8] Z. Zhang, Y.P. Handa, An In Situ Study of Plasticization of Polymers by High-Pressure Gases, J. Polym. Sci. Part B Polym. Phys. 36 (1997) 977-982. doi:10.1002/(SICI)1099-0488(19980430)36:6<977::AIDPOLB5>3.0.CO;2-D.

[9] H. Siddique, Y. Bhole, L.G. Peeva, A.G. Livingston, Pore preserving crosslinkers for polyimide OSN membranes, J. Memb. Sci. 465 (2014) 138-150. doi:10.1016/j.memsci.2014.03.031.

[10] K. Friess, J.C. Jansen, O. Vopička, A. Randová, V. Hynek, M. Šípek, L. Bartovská, P. Izák, M. Dingemans, J. Dewulf, H. Van Langenhove, E. Drioli, Comparative study of sorption and permeation techniques for the determination of heptane and toluene transport in polyethylene membranes, J. Memb. Sci. 338 (2009) 161-174. doi:10.1016/J.MEMSCI.2009.04.030.

[11] S.B. Harogoppad, T.M. Aminabhavi, Diffusion and Sorption of Organic Liquids through Polymer Membranes. 5. Neoprene, Styrene-Butadiene-Rubber, Ethylene-Propylene-Diene Terpolymer, and Natural Rubber versus Hydrocarbons (C8-C16), Macromolecules. 24 (1991) 2598-2605. 
doi:10.1021/ma00009a070.

[12] E. Favre, Q.T. Nguyen, P. Schaetzel, R. Clement, J. Neel, Sorption of organic solvents into dense silicone membranes. Part 1.-Validity and limitations of Flory-Huggins and related theories, J. Chem. Soc. Faraday Trans. 89 (1993) 4339-4346. doi:10.1039/FT9938904339.

[13] O. Vopička, A. Randová, K. Friess, Sorption of vapours and liquids in PDMS: novel data and analysis with the GAB model of multilayer adsorption, Eur. Polym. J. 60 (2014) 49-57. doi:10.1016/J.EURPOLYMJ.2014.08.009.

[14] A. Randová, L. Bartovská, Š. Hovorka, K. Friess, P. Izák, The membranes (Nafion and LDPE) in binary liquid mixtures benzene + methanol - sorption and swelling, Eur. Polym. J. 45 (2009) 2895-2901. doi:10.1016/J.EURPOLYMJ.2009.06.023.

[15] K. Spaeth, G. Kraus, G. Gauglitz, In-situ characterization of thin polymer films for applications in chemical sensing of volatile organic compounds by spectroscopic ellipsometry, Fresenius J. Anal. Chem. 357 (1997) 292-296. doi:10.1007/s002160050155.

[16] N.R. Horn, D.R. Paul, Carbon Dioxide Sorption and Plasticization of Thin Glassy Polymer Films Tracked by Optical Methods, Macromolecules. 45 (2012) 2820-2834. doi:Doi 10.1021/Ma300177k.

[17] D.S. Pope, W.J. Koros, H.B. Hopfenberg, Sorption and Dilation of Poly(1-(trimethylsilyl)-1-propyne) by Carbon Dioxide and Methane, Macromolecules. 27 (1994) 5839-5844. doi:10.1021/ma00098a043.

[18] B.W. Rowe, B.D. Freeman, D.R. Paul, Physical aging of ultrathin glassy polymer films tracked by gas permeability, Polymer (Guildf). 50 (2009) 5565-5575. doi:DOI: 10.1016/j.polymer.2009.09.037.

[19] N.R. Horn, D.R. Paul, Carbon dioxide plasticization and conditioning effects in thick vs. thin glassy polymer films, Polymer (Guildf). 52 (2011) 1619-1627. doi:DOI 10.1016/j.polymer.2011.02.007.

[20] S. Peter, H. Meyer, J. Baschnagel, Thickness-Dependent Reduction of the Glass-Transition Temperature in Thin Polymer Films with a Free Surface, J. Polym. Sci. Part B Polym. Phys. 44 (2006) 2951-2967. doi:10.1002/polb.

[21] M.D. Ediger, J.A. Forrest, Dynamics near Free Surfaces and the Glass Transition in Thin Polymer Films: A View to the Future, Macromolecules. 47 (2014) 471-478. doi:10.1021/ma4017696.

[22] Z. Fakhraai, J.A. Forrest, Measuring the Surface Dynamics of Glassy Polymers, Science (80-. ). 319 (2008) 600-604. doi:10.1126/science.1151205 ARTICLE.

[23] F. Buss, J. Gö, P. Scharfer, W. Schabel, From Micro to Nano Thin Polymer Layers: Thickness and Concentration Dependence of Sorption and the Solvent Diffusion Coefficient, Macromolecules. 48 (2015) 8285-8293. doi:10.1021/acs.macromol.5b01648.

[24] W. Ogieglo, H. Wormeester, M. Wessling, N.E. Benes, Probing the Surface Swelling in Ultra-Thin Supported Polystyrene Films During Case II Diffusion of n-Hexane, Macromol. Chem. Phys. 214 (2013) 2480-2488. doi:DOI 10.1002/macp.201300371.

[25] W. Ogieglo, H. Wormeester, M. Wessling, N.E. Benes, Temperature-induced transition of the diffusion mechanism of n-hexane in ultra-thin polystyrene films, resolved by in-situ Spectroscopic Ellipsometry, Polymer (Guildf). 54 (2013) 341-348. doi:DOI 10.1016/j.polymer.2012.11.038.

[26] J.S. Papanu, In Situ Ellipsometry to Monitor Swelling and Dissolution of Thin Polymer Films, J. Electrochem. Soc. 136 (1989) 1195. doi:10.1149/1.2096852.

[27] H. Elbs, G. Krausch, Ellipsometric determination of Flory-Huggins interaction parameters in solution, Polymer (Guildf). 45 (2004) 7935-7942. doi:10.1016/j.polymer.2004.09.021.

[28] K. Spaeth, G. Gauglitz, Characterisation of the optical properties of thin polymer films for their application in detection of volatile organic compounds, Mater. Sci. Eng. C. 5 (1998) 187-191. doi:10.1016/S0928-4931(97)00041-6.

[29] W.H. Brown, B.L. Iverson, E. Anslyn, C.S. Foote, Organic Chemistry, in: Org. Chem., 7th ed., Brooks/Cole, Cengage Learning, Belmont, 2014: pp. 341-400.

[30] W. Ogieglo, H. Wormeester, K.-J. Eichhorn, M. Wessling, N.E. Benes, In situ ellipsometry studies on swelling of thin polymer films: A review, Prog. Polym. Sci. (2014) 1-37. doi:10.1016/j.progpolymsci.2014.09.004.

[31] K. Chan, K.K. Gleason, Initiated Chemical Vapor Deposition of Linear and Cross-linked poly(2hydroxyethyl methacrylate) for Use as Thin-Film Hydrogels, Langmuir. (2005) 8930-8939. 
[32] W. Ogieglo, H. van der Werf, K. Tempelman, H. Wormeester, M. Wessling, A. Nijmeijer, N.E. Benes, n-Hexane induced swelling of thin PDMS films under non-equilibrium nanofiltration permeation conditions, resolved by spectroscopic ellipsometry, J. Memb. Sci. 437 (2013) 313-323. doi:10.1016/j.memsci.2013.04.039.

[33] S.M. Sirard, P.F. Green, K.P. Johnston, Spectroscopic Ellipsometry Investigation of the Swelling of Poly(Dimethylsiloxane) Thin Films with High Pressure Carbon Dioxide, J. Phys. Chem. B. 105 (2001) 766-772. doi:10.1021/jp002592d.

[34] K. Simons, K. Nijmeijer, J.G. Sala, H. van der Werf, N.E. Benes, T.J. Dingemans, M. Wessling, $\mathrm{CO}_{2}$ sorption and transport behavior of ODPA-based polyetherimide polymer films, Polymer (Guildf). 51 (2010) 3907-3917. doi:10.1016/j.polymer.2010.06.031.

[35] M.D. Miller, M.L. Bruening, R. V June, V. Re, M. Recei, V. August, Correlation of the Swelling and Permeability of Polyelectrolyte Multilayer Films, Chem. Mater. 17 (2005) 5375-5381.

[36] W. Ogieglo, H. Wormeester, M. Wessling, N.E. Benes, Effective medium approximations for penetrant sorption in glassy polymers accounting for excess free volume, Polymer (Guildf). 55 (2014) 1737-1744. doi:10.1016/j.polymer.2014.02.040.

[37] P.J. Flory, Principles of Polymer Chemistry, 1st Ed., Cornell University Press, New York, 1953.

[38] P.J. Flory, J.J. Rehner, Statistical Mechanics of Cross-Linked Polymer Networks II. Swelling, J. Chem. Phys. 11 (1943) 521-526. http://dx.doi.org/10.1063/1.1723792.

[39] A.A.R. W.J. Koros, D.R. Paul, Carbon dioxide sorption and transport in polycarbonate, J. Polym. Sci. Part B Polym. Physics1. 14 (1976) 687-702. doi:10.1002/pol.1976.180140410.

[40] W.J. Koros, a. H. Chan, D.R. Paul, Sorption and transport of various gases in polycarbonate, J. Memb. Sci. 2 (1977) 165-190. doi:10.1016/S0376-7388(00)83242-1.

[41] N.A. Neuburger, B.E. Eichinger, Critical experimental test of the Flory-Rehner theory of swelling, Macromolecules. 21 (1988) 3060-3070. doi:10.1021/ma00188a026.

[42] M.G. Bawendi, K.F. Freed, Systematic corrections to Flory-Huggins theory: Polymer-solvent-void systems and binary blend-void systems, J. Chem. Phys. 88 (1988) 2741-2756. doi:doi:http://dx.doi.org/10.1063/1.454005.

[43] A. Holtzer, Does Flory-Huggins theory help in interpreting solute partitioning experiments?, Biopolymers. 34 (1994) 315-320. doi:10.1002/bip.360340303.

[44] B. Hancock, G. Zografi, The Use of Solution Theories for Predicting Water Vapor Absorption by Amorphous Pharmaceutical Solids: A Test of the Flory-Huggins and Vrentas Models, Pharm. Res. 10 (1993) 1262-1267. doi:10.1023/A:1018901325842.

[45] T.-S. Chung, The limitations of using Flory-Huggins equation for the states of solutions during asymmetric hollow-fiber formation, J. Memb. Sci. $126 \quad$ (1997) 19-34. doi:http://dx.doi.org/10.1016/S0376-7388(96)00269-4.

[46] A.I. Pesci, K.F. Freed, Lattice theory of polymer blends and liquid mixtures: Beyond the Flory-Huggins approximation, J. Chem. Phys. 90 (1989) 2017-2026. doi:doi:http://dx.doi.org/10.1063/1.456044.

[47] I.C. Sanchez, R.H. Lacombe, Statistical Thermodynamics of Polymer Solutions, Macromolecules. 11 (1978) 1145-1156. doi:10.1021/ma60066a017.

[48] F. Doghieri, G.C. Sarti, Nonequilibrium Lattice Fluids: A Predictive Model for the Solubility in Glassy Polymers, Macromolecules. 29 (1996) 7885-7896. doi:10.1021/ma951366c.

[49] J.S. Vrentas, C.M. Vrentas, Integral sorption in glassy polymers, Chem. Eng. Sci. 53 (1998) 629-638. doi:http://dx.doi.org/10.1016/S0009-2509(97)00368-0.

[50] J.S. Vrentas, C.M. Vrentas, Hysteresis Effects for Sorption in Glassy Polymers, Macromolecules. 29 (1996) 4391-4396. doi:10.1021/ma9509691.

[51] J.S. Vrentas, C.M. Vrentas, Sorption in Glassy Polymers, Macromolecules. 24 (1991) $2404-2412$. doi:10.1016/S0009-2509(97)00368-0.

[52] L. Leibler, K. Sekimoto, On the sorption of gases and liquids in glassy polymers, Macromolecules. 26 (1993) 6937-6939. doi:10.1021/ma00077a034.

[53] A.R. Berens, H.B. Hopfenberg, Diffusion and relaxation in glassy polymer powders: 2. Separation of diffusion and relaxation parameters, Polymer (Guildf). 19 (1977) 489-496. doi:Doi 10.1016/0032- 
3861(78)90269-0.

[54] V. Carla, K. Wang, Y. Hussain, K. Efimenko, J. Genzer, C. Grant, G.C. Sarti, R.G. Carbonell, F. Doghieri, Nonequilibrium model for sorption and swelling of bulk glassy polymer films with Supercritical carbon dioxide, Macromolecules. 38 (2005) 10299-10313. doi:Doi 10.1021/Ma0506684.

[55] J.Q. Pham, K.P. Johnston, P.F. Green, Retrograde Vitrification in CO2/Polystyrene Thin Films, J. Phys. Chem. B. 108 (2004) 3457-3461. doi:10.1021/Jp0367651.

[56] J. Pham, S. Sirard, K. Johnston, P. Green, Pressure, Temperature, and Thickness Dependence of CO2Induced Devitrification of Polymer Films, Phys. Rev. Lett. 91 (2003) 175503. doi:10.1103/PhysRevLett.91.175503.

[57] B.W. Rowe, B.D. Freeman, D.R. Paul, Influence of previous history on physical aging in thin glassy polymer films as gas separation membranes, Polymer (Guildf). 51 (2010) 3784-3792. doi:DOI: 10.1016/j.polymer.2010.06.004.

[58] D. Turnbull, M.H. Cohen, Free-Volume Model of the Amorphous Phase: Glass Transition, J. Chem. Phys. 34 (1961) 120. doi:10.1063/1.1731549.

[59] B. a. Miller-Chou, J.L. Koenig, A review of polymer dissolution, Prog. Polym. Sci. 28 (2003) 12231270. doi:10.1016/S0079-6700(03)00045-5.

[60] E.J. Kappert, M.J.T. Raaijmakers, W. Ogieglo, A. Nijmeijer, C. Huiskes, N.E. Benes, Temperature calibration procedure for thin film substrates for thermo-ellipsometric analysis using melting point standards, Thermochim. Acta. 601 (2015) 29-32. doi:10.1016/j.tca.2014.12.017.

[61] C.M. Herzinger, B. Johs, W. a. McGahan, J. a. Woollam, W. Paulson, Ellipsometric determination of optical constants for silicon and thermally grown silicon dioxide via a multi-sample, multi-wavelength, multi-angle investigation, J. Appl. Phys. 83 (1998) 3323. doi:10.1063/1.367101.

[62] K. Tempelman, E.J. Kappert, M.J.T. Raaijmakers, H. Wormeester, N.E. Benes, Dealing with inaccuracies in the analysis on solvent-induced swelling of transparent thin films using in situ spectroscopic ellipsometry in the visible wavelength range, Surf. Interface Anal. 49 (2016) 538-547. doi:10.1002/sia.6191.

[63] G.M. Whitesides, The origins and the future of microfluidics., Nature. 442 (2006) 368-73. doi:10.1038/nature05058.

[64] J.M.S. Henis, M.K. Tripodi, A novel approach to gas separations using composite hollow fiber membranes, Sep. Sci. Technol. 15 (1980) 1059-1063. doi:10.1080/01496398008076287.

[65] J.G.A. Bitter, J.P. Haan, H.C. Rijkens, Process for the separation of solvents from hydrocarbons dissolved in the solvents, US4748288, 1988.

[66] J.N. Lee, C. Park, G.M. Whitesides, Solvent compatibility of poly(dimethylsiloxane)-based microfluidic devices., Anal. Chem. 75 (2003) 6544-54. doi:10.1021/ac0346712.

[67] A. Dobrak-Van Berlo, I.F.J. Vankelecom, B. Van der Bruggen, Parameters determining transport mechanisms through unfilled and silicalite filled PDMS-based membranes and dense PI membranes in solvent resistant nanofiltration: Comparison with pervaporation, J. Memb. Sci. 374 (2011) 138-149. doi:10.1016/j.memsci.2011.03.027.

[68] L. Leitner, C. Harscoat-Schiavo, C. Vallières, Experimental contribution to the understanding of transport through polydimethylsiloxanenanofiltration membranes: Influence of swelling, compaction and solvent on permeation properties, Polym. Test. 33 (2014) 88-96. doi:10.1016/j.polymertesting.2013.10.016.

[69] L.E.M. Gevers, G. Meyen, K. De Smet, P. Van De Velde, F. Du Prez, I.F.J. Vankelecom, P. a. Jacobs, K. De Smet, L.E.M. Gevers, A. Livingston, D. Nair, S. Aerts, S. Kuypers, P. a. Jacobs, Physico-chemical interpretation of the SRNF transport mechanism for solvents through dense silicone membranes, J. Memb. Sci. 274 (2004) 173-182. doi:10.1016/j.memsci.2003.11.007.

[70] D.R. Machado, D. Hasson, R. Semiat, Effect of solvent properties on permeate flow through nanofiltration membranes. Part I: investigation of parameters affecting solvent flux, J. Memb. Sci. 163 (1999) 93-102. doi:10.1016/S0376-7388(99)00158-1.

[71] E. Favre, Swelling of crosslinked polydimethylsiloxane networks by pure solvents: influence of temperature, Eur. Polym. J. 32 (1996) 1183-1188.

[72] J.H. Gibbs, E. a. DiMarzio, Nature of the Glass Transition and the Glassy State, J. Chem. Phys. 28 
(1958) 373. doi:10.1063/1.1744141.

[73] K. Haraya, K. Obata, T. Hakuta, H. Yoshitome, Performance of Gas Separator with High-Flux Polyimide Hollow Fiber Membrane, Sep. Sci. Technol. 23 (1988) 305-319.

[74] M.E. Rezac, W.J. Koros, S.J. Miller, Membrane-assisted dehydrogenation of n-butane Influence of membrane properties on system performance, J. Memb. Sci. 93 (1994) 193-201. doi:10.1016/03767388(94)80007-3.

[75] L.S. White, A.R. Nitsch, Solvent recovery from lube oil filtrates with a polyimide membrane, J. Memb. Sci. 179 (2000) 267-274. doi:10.1016/S0376-7388(00)00517-2.

[76] M. Lupión, F.J. Gutiérrez Ortiz, B. Navarrete, V.J. Cortés, Assessment performance of high-temperature filtering elements, Fuel. 89 (2010) 848-854. doi:10.1016/j.fuel.2009.04.016.

[77] L.S. White, C.R. Wildemuth, Aromatics Enrichment in Refinery Streams Using Hyperfiltration, Ind. Eng. Chem. Res. 45 (2006) 9136-9143.

[78] H. Werhan, A. Farshori, P. Rudolf von Rohr, Separation of lignin oxidation products by organic solvent nanofiltration, J. Memb. Sci. 423-424 (2012) 404-412. doi:10.1016/j.memsci.2012.08.037.

[79] K. Vanherck, G. Koeckelberghs, I.F.J. Vankelecom, Crosslinking polyimides for membrane applications: A review, Prog. Polym. Sci. 38 (2013) 874-896. doi:10.1016/j.progpolymsci.2012.11.001.

[80] M.A.M. Beerlage, Polyimide ultrafiltration membranes for non-aqueous systems, University of Twente, 1994.

[81] X. Qiao, T. Chung, Fundamental Characteristics of Sorption, Swelling, and Permeation of P84 Copolyimide Membranes for Pervaporation Dehydration of Alcohols, Ind. Eng. Chem. Res. 15 (2005) 8938-8943.

[82] I. Soroko, M.P. Lopes, A. Livingston, The effect of membrane formation parameters on performance of polyimide membranes for organic solvent nanofiltration (OSN): Part A. Effect of polymer/solvent/nonsolvent system choice, J. Memb. Sci. 381 (2011) 152-162. doi:10.1016/j.memsci.2011.07.027.

[83] H. Qariouh, R. Schué, F. Schué, C. Bailly, Sorption, diffusion and pervaporation of water/ethanol mixtures in polyetherimide membranes, Polym. Int. 48 (1999) 171-180. doi:10.1002/(SICI)10970126(199903)48:3<171::AID-PI126>3.0.CO;2-W.

[84] P. Vandezande, X. Li, L.E.M. Gevers, I.F.J. Vankelecom, High throughput study of phase inversion parameters for polyimide-based SRNF membranes, J. Memb. Sci. 330 (2009) 307-318. doi:10.1016/j.memsci.2008.12.068.

[85] L. Lin, S.A. Bistrup, Processing effects on optical properties of spin coated polyimide films, J. Appl. Polym. Sci. 49 (1993) 1277-1289.

[86] B. Bolto, M. Hoang, Z. Xie, A review of water recovery by vapour permeation through membranes., Water Res. 46 (2012) 259-66. doi:10.1016/j.watres.2011.10.052.

[87] J.H. Kim, S.Y. Ha, Y.M. Lee, Gas permeation of poly(amide-6-b-ethylene oxide) copolymer, J. Membr. 190 (2001) 179-193.

[88] H.J. Zwijnenberg, a. M. Krosse, K. Ebert, K. V. Peinemann, F.P. Cuperus, Acetone-stable nanofiltration membranes in deacidifying vegetable oil, J. Am. Oil Chem. Soc. 76 (1999) 83-87. doi:10.1007/s11746999-0051-1.

[89] T. Masuda, E. Isobe, T. Higashimura, Poly[1-(trimethylsily1)-1-propyne]: A New High Polymer Synthesized with Transition-Metal Catalysts and Characterized by Extremely High Gas Permeability, J. Am. Chem. Soc. 105 (1983) 7473-7474.

[90] G. Consolati, I. Genco, M. Pegoraro, L. Zanderighi, Positron Annihilation lifetime (PAL) in Poly [1(trimethyl-silyl)propine] (PTMSP): Free Volume Determination and Time Dependence of Permeability, J. Polym. Sci. Part B Polym. Phys. 34 (1996) 357-367.

[91] C.H. Lau, P.T. Nguyen, M.R. Hill, A.W. Thornton, K. Konstas, C.M. Doherty, R.J. Mulder, L. Bourgeois, A.C.Y. Liu, D.J. Sprouster, J.P. Sullivan, T.J. Bastow, A.J. Hill, D.L. Gin, R.D. Noble, Ending Aging in Super Glassy Polymer Membranes, Angew. Chemie. 126 (2014) 5426-5430. doi:10.1002/ange.201402234.

[92] A.V. Volkov, D.F. Stamatialis, V.S. Khotimsky, V.V. Volkov, M. Wessling, N.A. Platé, Poly[1(trimethylsilyl)-1-propyne] as a solvent resistance nanofiltration membrane material, J. Memb. Sci. 281 (2006) 351-357. doi:10.1016/j.memsci.2006.04.004. 
[93] A.V. Volkov, V.V. Parashchuk, D.F. Stamatialis, V.S. Khotimsky, V.V. Volkov, M. Wessling, High permeable PTMSP/PAN composite membranes for solvent nanofiltration, J. Memb. Sci. 333 (2009) 8893. doi:10.1016/j.memsci.2009.01.050.

[94] I. Pinnau, L.G. Toy, Transport of organic vapors through poly(1-trimethylsilyl-1-propyne), J. Memb. Sci. 116 (1996) 199-209. doi:10.1016/0376-7388(96)00041-5.

[95] N.A. Hoenich, C. Woffindin, A. Brennan, P.J. Cox, J.N.S. Matthews, M. Goldfinch, A Comparison of Three Brands of Polysulfone Membranes, J. Am. Soc. Nephrol. 7 (1996) 871-876.

[96] K. Majewska-Nowak, Synthesis and properties of polysulfone membranes, Desalination. 71 (1989) 8395. doi:10.1016/0011-9164(89)80001-3.

[97] M. Rabiller-Baudry, M. Le Maux, B. Chaufer, L. Begoin, Characterisation of cleaned and fouled membrane by ATR-FTIR and EDX analysis coupled with SEM: Application to UF of skimmed milk with a PES membrane, Desalination. 146 (2002) 123-128. doi:10.1016/S0011-9164(02)00503-9.

[98] Y. Li, L.H. Wee, A. Volodin, J.A. Martens, I.F.J. Vankelecom, Polymer supported ZIF-8 membranes prepared via an interfacial synthesis method, Chem. Commun. 51 (2015) 918-920. doi:10.1039/C4CC06699E.

[99] J. Geens, B. Van der Bruggen, C. Vandecasteele, Transport model for solvent permeation through nanofiltration membranes, Sep. Purif. Technol. 48 (2006) 255-263. doi:10.1016/J.SEPPUR.2005.07.032.

[100] R.J. Petersen, Composite reverse osmosis and nanofiltration membranes, J. Memb. Sci. 83 (1993) 81150. doi:10.1016/0376-7388(93)80014-O.

[101] A.K. Hołda, B. Aernouts, W. Saeys, I.F.J. Vankelecom, Study of polymer concentration and evaporation time as phase inversion parameters for polysulfone-based SRNF membranes, J. Memb. Sci. 442 (2013) 196-205. doi:10.1016/J.MEMSCI.2013.04.017.

[102] M. Cheryan, Ultrafiltration and Microfiltration Handbook, CRC Press, Taylor \& Francis Group, 1998.

[103] W.J. Lau, A.F. Ismail, N. Misdan, M.A. Kassim, A recent progress in thin film composite membrane: A review, Desalination. 287 (2012) 190-199. doi:10.1016/j.desal.2011.04.004.

[104] S. Darvishmanesh, J.C. Jansen, F. Tasselli, E. Tocci, P. Luis, J. Degrève, E. Drioli, B. Van der Bruggen, Novel polyphenylsulfone membrane for potential use in solvent nanofiltration, J. Memb. Sci. 379 (2011) 60-68. doi:10.1016/J.MEMSCI.2011.05.045.

[105] N. Scharnagl, H. Buschatz, Polyacrylonitrile (PAN) membranes for ultra- and microfiltration, Desalination. 139 (2001) 191-198.

[106] J. Wang, Z. Yue, J.S. Ince, J. Economy, Preparation of nanofiltration membranes from polyacrylonitrile ultrafiltration membranes, J. Memb. Sci. 286 (2006) 333-341. doi:10.1016/J.MEMSCI.2006.10.022.

[107] T.D. Tran, S. Mori, M. Suzuki, Plasma modification of polyacrylonitrile ultrafiltration membrane, Thin Solid Films. 515 (2007) 4148-4152. doi:10.1016/J.TSF.2006.02.045.

[108] H.R. Lohokare, S.C. Kumbharkar, Y.S. Bhole, U.K. Kharul, Surface modification of polyacrylonitrile based ultrafiltration membrane, J. Appl. Polym. Sci. 101 (2006) 4378-4385. doi:10.1002/app.23917.

[109] N.-W. Oh, J. Jegal, K.-H. Lee, Preparation and characterization of nanofiltration composite membranes using polyacrylonitrile (PAN). I. preparation and modification of PAN supports, J. Appl. Polym. Sci. 80 (2001) 1854-1862. doi:10.1002/app.1282. 